Article

\title{
Human Peripheral Blood-Derived Endothelial Colony-Forming Cells Are Highly Similar to Mature Vascular Endothelial Cells yet Demonstrate a Transitional Transcriptomic Signature
}

\author{
Anton G. Kutikhin ${ }^{1, *,+}$, Alexey E. Tupikin ${ }^{2,+}{ }^{+}$, Vera G. Matveeva ${ }^{1}{ }^{\mathbb{C}}$, Daria K. Shishkova ${ }^{1}$, \\ Larisa V. Antonova ${ }^{1}\left(\mathbb{D}\right.$, Marsel R. Kabilov ${ }^{2}(\mathbb{D})$ and Elena A. Velikanova ${ }^{1}$ \\ 1 Research Institute for Complex Issues of Cardiovascular Diseases, 6 Sosnovy Boulevard, Kemerovo 650002, \\ Russia; matveeva_vg@mail.ru (V.G.M.); shidk@kemcardio.ru (D.K.S.); antonova.la@mail.ru (L.V.A.); \\ telella@mail.ru (E.A.V.) \\ 2 Institute of Chemical Biology and Fundamental Medicine, Siberian Branch of the Russian Academy of \\ Sciences, 8 Lavrentiev Avenue, Novosibirsk 630090, Russia; alenare@niboch.nsc.ru (A.E.T.); \\ kabilov@niboch.nsc.ru (M.R.K.) \\ * Correspondence: antonkutikhin@gmail.com; Tel.: +7-960-907-70-67 \\ + These authors equally contributed to this work.
}

Received: 2 March 2020; Accepted: 2 April 2020; Published: 3 April 2020

check for updates

\begin{abstract}
Endothelial colony-forming cells (ECFC) are currently considered as a promising cell population for the pre-endothelialization or pre-vascularization of tissue-engineered constructs, including small-diameter biodegradable vascular grafts. However, the extent of heterogeneity between ECFC and mature vascular endothelial cells (EC) is unclear. Here, we performed a transcriptome-wide study to compare gene expression profiles of ECFC, human coronary artery endothelial cells (HCAEC), and human umbilical vein endothelial cells (HUVEC). Characterization of the abovementioned cell populations was carried out by immunophenotyping, tube formation assay, and evaluation of proliferation capability while global gene expression profiling was conducted by means of RNA-seq. ECFC were similar to HUVEC in terms of immunophenotype $\left(\mathrm{CD} 31^{+} \mathrm{vWF}^{+} \mathrm{KDR}^{+} \mathrm{CD} 146^{+} \mathrm{CD} 34^{-} \mathrm{CD} 133^{-} \mathrm{CD} 45^{-} \mathrm{CD} 90^{-}\right)$and tube formation activity yet had expectedly higher proliferative potential. HCAEC and HUVEC were generally similar to ECFC with regards to their global gene expression profile; nevertheless, ECFC overexpressed specific markers of all endothelial lineages (NRP2, NOTCH4, LYVE1), in particular lymphatic EC (LYVE1), and had upregulated extracellular matrix and basement membrane genes (COL1A1, COL1A2, COL4A1, $C O L 4 A 2)$. Proteomic profiling for endothelial lineage markers and angiogenic molecules generally confirmed RNA-seq results, indicating ECFC as an intermediate population between HCAEC and HUVEC. Therefore, gene expression profile and behavior of ECFC suggest their potential to be applied for a pre-endothelialization of bioartificial vascular grafts, whereas in terms of endothelial hierarchy they differ from HCAEC and HUVEC, having a transitional phenotype.
\end{abstract}

Keywords: endothelial colony-forming cells; peripheral blood mononuclear cells; coronary artery endothelial cells; umbilical vein endothelial cells; adipose tissue-derived stromal vascular fraction; endothelial lineages; gene expression; RNA-seq; transcriptome profiling; transcriptomic signatures

\section{Introduction}

Coronary artery bypass graft surgery remains an efficient and widespread surgical option to treat coronary artery disease [1], yet availability of autologous conduits such as internal mammary arteries 
or saphenous veins is often limited in patients with widespread atherosclerotic vascular disease or in those whose vessels are anatomically incompatible or have already been harvested for a previous procedure [2-4]. Allogeneic and xenogeneic blood vessels demonstrate only a limited efficiency due to the risk of transmissible disease, graft-versus-host disease, infection, and calcification $[4,5]$ while prosthetic grafts fabricated from biostable synthetic polymers such as poly(ethylene terephthalate), expanded poly(tetrafluoroethylene), and polyurethanes show inferior patency rates in small-diameter applications due to poor endothelialization, low blood flow, and compliance mismatch, all resulting in intimal hyperplasia, thrombosis, or (pseudo)aneurysms [2-5]. In addition, both xenogeneic and biostable synthetic vascular conduits lack growth adaptation potential and therefore demand repeated surgery and ultimately lead to unacceptable long-term outcomes [5,6]. Hence, vascular tissue engineering has emerged as one of the most promising approaches for producing mechanically competent and biocompatible small-diameter vascular substitutes [2,4,7,8]. This frequently involves the use of biodegradable polymers to provide a tubular scaffold for cell adhesion, proliferation, and intramural migration followed by the de novo formation of the vascular tissue and subsequent scaffold degradation [8].

The major advantage of the biodegradable vascular grafts over biostable ones is better endothelialization, a process pivotal for the prevention of thrombotic occlusion [9-11]. However, thrombosis generally occurs within the first minutes or hours upon contact of the blood with the polymer surface, negating improved biocompatibility of biodegradable polymers. Hence, pre-endothelialization is strongly required to secure zero thrombogenicity of the vascular graft and screening of pertinent endothelial cell sources is rapidly ongoing. Recent studies report successful differentiation of peripheral blood mononuclear cells (PBMC) into endothelial colony-forming cells (ECFC) [12,13] which is particularly efficient after percutaneous coronary intervention due to a mechanical injury provoking the release of ECFC precursors into the bloodstream [14]. ECFC exhibit high angiogenic [15,16] and proliferative [16] capability and can be suggested as an appropriate cell population for the endothelialization of the tubular scaffolds prior to implantation.

In contrast to short-term patency which is largely determined by endothelial integrity, long-term patency depends on endothelial homeostasis maintained by a proper gene expression profile. Distinct endothelial lineages have considerable differences regarding their transcriptome, and the extent of endothelial cell heterogeneity is currently a matter of debate [17-19]. Nevertheless, endothelial identity is mandatory to control vascular tone and for the paracrine regulation of vascular specification within the graft [20-22]. Accordingly, transcriptomic signatures of pre-seeded endothelial cells (EC) should correspond to those of mature resident EC.

To assess whether ECFC are suitable for in vitro pre-endothelialization of vascular grafts, we compared the global gene expression profile of human PBMC-derived ECFC in comparison with human coronary artery endothelial cells (HCAEC), human umbilical vein endothelial cells (HUVEC), and human subcutaneous adipose tissue-derived stromal vascular fraction (SAT-SVF) by means of RNA-sequencing (RNA-seq). We found that the baseline gene expression profile of ECFC is close to that of HCAEC and HUVEC but expectedly different from SAT-SVF, testifying to their utility for the seeding of tubular scaffolds before implantation to improve their short- and long-term performance.

\section{Materials and Methods}

\subsection{Cell Culture}

Peripheral blood (20 mL) was withdrawn from 8 male patients during percutaneous coronary intervention performed in the Research Institute for Complex Issues of Cardiovascular Diseases (Kemerovo, Russian Federation). The study design was approved by the Local Ethical Committee (ID 657460, approved 28 October 2016). All patients provided written informed consent before the recruitment after receiving a full explanation of the study. A complete blood count was performed utilizing an automated hematology analyzer (MEK8222K, Nihon Kohden, Tokyo, Japan). Average 
white blood cell count was $6.8 \times 10^{9} / \mathrm{L}\left(13.6 \times 10^{7}\right.$ in $\left.20 \mathrm{~mL}\right)$. PBMC were isolated using Histopaque density media 1077 (10771, Sigma, St. Louis, MO, USA) according to the manufacturer's instructions. The cells obtained from the interphase were washed twice with phosphate buffered saline (PBS, 70011044, Thermo Fisher Scientific, MA, USA) followed by centrifugation. We performed isolation and enrichment of ECFC using the modified protocol by Kolbe et al. [23]. Briefly, cells ( $2.8 \times 10^{7}$ in total) were resuspended in the EGM-2MV (CC-3202, Lonza, Basel, Switzerland) medium supplemented with $5 \%$ fetal bovine serum (FBS) (SH3007103, HyClone) and seeded into the collagen-coated $25 \mathrm{~cm}^{2}$ flasks (356484, Corning, New York, NY, USA). In the first two days, the medium was changed daily to remove non-adherent cells and debris, and then was changed three times a week. After one week of culture, the cells were dissociated with trypsin and reseeded to fibronectin-coated $75 \mathrm{~cm}^{2}$ flasks (354521, Corning). The frequency of ECFC at the time of reseeding was 1 per $10^{7}$ cultured PBMC ( 3 or 4 colonies per $75 \mathrm{~cm}^{2}$ flask). Passaging was performed at 70\%-80\% confluence. Immunophenotyping and functional assays were performed at the 19th-22nd day of culture (average ECFC yield $1.5 \times$ $10^{6}-2.0 \times 10^{6}$ cells per $75 \mathrm{~cm}^{2}$ flask). Cells were counted after the trypsinization using an automated cell counter (LUNA-II, Logos Biosystems, Seoul, South Korea).

SAT $\left(1.7-2.0 \mathrm{~cm}^{3}\right)$ was harvested from the same patients, washed in Hank's balanced salt solution (HBSS, 14185052, Gibco), minced, and treated with $0.2 \%$ collagenase type I (17100017, Gibco) during 30 minutes at $37{ }^{\circ} \mathrm{C}$. Enzymatic digestion was stopped by $10 \%$ FBS. Dissociated tissue was then filtered $(100 \mu \mathrm{m})$ and centrifuged at $1000 \times \mathrm{g}$. Cell pellet was resuspended in EGM-2MV, seeded into collagen-coated $25 \mathrm{~cm}^{2}$ flasks (354484, Corning) and then cultured as above.

HUVEC were isolated from umbilical cords according to the modified protocol of Jaffe et al. [24], while primary HCAEC were purchased from Cell Applications (San Diego, CA, USA) (300K-05a). For the culture of HUVEC and HCAEC, we used EGM-2MV (CC-3202, Lonza) medium supplemented with 5\% FBS (SH3007103, HyClone) similar to the culture of ECFC and SAT-SVF. Initially, we seeded $0.5 \times 10^{6}$ cells per collagen-coated $25 \mathrm{~cm}^{2}$ flask (356484, Corning). At the next passage, HUVEC and HCAEC were reseeded into fibronectin-coated $75 \mathrm{~cm}^{2}$ flasks (354521, Corning). For all analyses, we collected HUVEC and HCAEC of the same passage and in the similar amount as ECFC and SAT-SVF (passage 3 or 4 , average yield $1.5 \times 10^{6}-2.0 \times 10^{6}$ cells per $75 \mathrm{~cm}^{2}$ flask).

All cell lines were cultured at standard cell culture conditions, and cell culture procedures were carried out under strict aseptic conditions. Visual control of culture growth was performed daily.

\subsection{Immunophenotyping}

A total of $100 \mu \mathrm{L}$ containing $0.5 \times 10^{5}-1.0 \times 10^{5}$ ECFC, HUVEC, or SAT-SVF removed from the plates and washed with PBS were taken for the staining with conjugated monoclonal antibodies against human antigens: (1) allophycocyanin (APC)-conjugated anti-CD31 (303116, BioLegend, San Diego, CA, USA, 1:60 dilution); (2) fluorescein isothiocyanate (FITC)-conjugated anti-von Willebrand factor (vWF, ab8822, Abcam, Cambridge, UK, 1:100); (3) phycoerythrin (PE)-conjugated anti-kinase insert domain receptor (KDR, 560494, BD Biosciences, San Jose, CA, USA, 1:50); (4) phycoerythrin-cyanine 7 (PE/Cy7)-conjugated anti-CD146 (361008, BioLegend, 1:60); (first panel); (5) FITC-conjugated anti-CD34 (343504, BioLegend, 1:60); (6) APC-conjugated anti-CD133 (372806, BioLegend, 1:60); (7) Krome Orange (KrO)-conjugated anti-CD45 (A96416, Beckman Coulter, 1:50); (second panel); (8) FITC-conjugated anti-CD90 (328108, BioLegend, 1:60); (9) APC/Cy7-conjugated anti-CD73 (344022, BioLegend, 1:60) (third panel). Fixation and permeabilization of the cells were performed using the IntraPrep Permeabilization Reagent (A07803, Beckman Coulter, Brea, CA, USA) when staining intracellular vWF. Samples were then resuspended in PBS and analyzed on a CytoFlex flow cytometer with CytExpert software (Beckman Coulter).

To verify flow cytometry results, the cells were cultured on fibronectin-coated coverslips and stained for characteristic endothelial markers CD31 and vWF. Briefly, the cells were fixed for $10 \mathrm{~min}$ in $4 \%$ paraformaldehyde (P6148, Sigma). To stain intracellular markers (vWF), permeabilization of the cells was additionally performed by treatment with $0.1 \%$ Triton X-100 (T8787, Sigma) for $15 \mathrm{~min}$. To 
block non-specific binding, the cells were treated with 1\% BSA (A9418, Sigma) for $1 \mathrm{~h}$. Samples were then incubated with primary antibodies (anti-CD31, ab119339, Abcam, 1:100) overnight at $4{ }^{\circ} \mathrm{C}$ and further with secondary antibodies (Alexa Fluor 568-conjugated goat anti-mouse, ab175473, Abcam, 1:600) and sheep FITC-conjugated anti-vWF antibody (ab8822, Abcam, 1:100). Control samples were mock-stained with $1 \%$ BSA instead of primary antibodies with all other procedures according to the protocol. Nuclei were counterstained with 4',6-diamidino-2-phenylindole (DAPI) (D9542, Sigma) at a concentration of $10 \mu \mathrm{g} / \mathrm{mL}$. Samples were mounted using ProLong Gold Antifade (P36930, Invitrogen) medium. Examination was carried out by confocal microscopy (LSM 700, Carl Zeiss, Oberkochen, Germany).

\subsection{Functional Assays}

For the evaluation of ECFC, HUVEC, and SAT-SVF tube-forming capacity, $200 \mu \mathrm{L}$ Matrigel (354234, Corning) was added to each well of a 24-well plate for $30 \mathrm{~min}$ at room temperature to allow polymerization. Cells were seeded at $1.0 \times 10^{5}$ cells per well and resuspended in EGM-2MV supplemented with $5 \%$ FBS. The formation of capillary-like tubes was monitored after $16 \mathrm{~h}$ by phase contrast microscopy (AxioObserver.A1, Carl Zeiss). Semi-quantitative image analysis (5 representative images per cell line) was performed employing WimTube (Wimasis, Cordoba, Spain).

To assess acetylated low-density lipoprotein cholesterol (acLDL) uptake and Ulex Europaeus agglutinin 1 (UEA) binding by ECFC and HUVEC, a total of $2.4 \mu \mathrm{g} / \mathrm{mL}$ 1,1'-dioctadecyl-3,3,3' $3^{\prime}{ }^{\prime}$-tetramethylindocarbocyanine perchlorate (DiI)-labeled acLDL (L3484, Invitrogen) was added to the cells with the following incubation for $2 \mathrm{~h}$ at $37^{\circ} \mathrm{C}$. The cells were then fixed with $2 \%$ paraformaldehyde for $15 \mathrm{~min}$ and incubated for $1 \mathrm{~h}$ with FITC-conjugated UEA (L9006, Sigma) at a concentration of $10 \mu \mathrm{g} / \mathrm{mL}$. Nuclei were counterstained with DAPI $(1.5 \mu \mathrm{g} / \mathrm{mL})$. Samples were mounted (ProLong Gold Antifade) and assessed utilizing confocal microscopy (10 representative fields of view per cell line).

Fluorescent staining of dividing nuclei in ECFC and HUVEC was performed using the Click-iT Plus EdU Alexa Fluor 488 Imaging Kit (C10637, Invitrogen) according to the manufacturer's protocol. The EdU exposure time was $6 \mathrm{~h}$. The samples were counterstained with DAPI $(10 \mu \mathrm{g} / \mathrm{mL})$, mounted (ProLong Gold Antifade), and analyzed using confocal microscopy. Positive cells (green) were counted in 10 representative fields of view, and then their ratio to the total number of cells in these fields was calculated.

The proliferative activity of ECFC and HUVEC (passage 4) was additionally evaluated by non-invasive electrical impedance monitoring (xCELLigence Real-Time Cell Analyzer Dual-Plate, ACEA Biosciences). Cells were seeded into 16-well E-plates (2801032, ACEA Biosciences, $2 \times 10^{4}$ cells per well) in duplicate, and impedance was measured over $100 \mathrm{~h}$. Cell-free culture medium was applied as a blank. Proliferation capability was defined as cell index doubling time calculated automatically by the instrument software.

\subsection{RNA-Seq}

RNA-seq was performed in SB RAS Genomics Core Facility (ICBFM SB RAS, Novosibirsk). Upon the withdrawal of culture medium and washing in ice-cold phosphate buffered saline (four $75 \mathrm{~cm}^{2}$ flasks per group), cells were lysed with TRIzol (15596018, Invitrogen) with the following total RNA isolation (Purelink RNA Micro Scale Kit, 12183016, Invitrogen) and DNAse treatment (DNASE70, Sigma). RNA integrity index (RIN) was assessed using RNA 6000 Pico Kit (5067-1513, Agilent) and Bioanalyzer 2100 (Agilent) (Figure S1 and Table S1) while RNA quantification was carried out using NanoDrop 2000 (Thermo Scientific) and Qubit 4 (Invitrogen). For the $1 \mu \mathrm{g}$ of isolated RNA, we performed rRNA depletion (RiboCop rRNA Depletion Kit V1.2, 037.96, Lexogen) followed by DNA library preparation (SENSE Total RNA-Seq Library Prep Kit, 042.96, Lexogen) and quality control (High Sensitivity DNA Kit, 5067-4626, Agilent) (Figure S2). DNA libraries were then quantified by 
qPCR (CFX96 Touch, Bio-Rad), pooled in equimolar amounts and sequenced (HiSeq 2000, Illumina) using $2 \times 132$ bp chemistry.

SENSE Total RNA-Seq uses a 9-nt-long random sequence of the starter and a 6-nt-long random sequence of the stopper hybridized to the RNA template. Therefore, it removed the first nine nucleotides from read 1 and the first six nucleotides from read 2 by cutadapt v.1.18. After the filtration of cut reads by quality $(\mathrm{QV}>20)$ and length $(>20)$, we performed an adapter trimming by TrimGalore v.0.4.4. The average number of reads exceeded 10,000,000. Read mapping to the human genome (hg38 with Ensembl annotation v.38.93) was conducted using CLC GW 12.0 (Qiagen) according to the following parameters: similarity fraction $=0.8$, length fraction $=0.8$, mismatch cost $=2$, insertion cost $=3$, deletion cost $=3$ (Table S1). To define differentially expressed genes (DEGs), we used multifactorial statistical analysis (CLC GW 12.0) based on the negative binomial regression.

The data reported in this study have been deposited in NCBI's Gene Expression Omnibus and are accessible through GEO Series accession number GSE131995 (https://www.ncbi.nlm.nih.gov/geo/ query/acc.cgi?acc=GSE131995).

\subsection{Proteomic Profiling}

Validation of RNA-seq was performed by means of dot blotting and conventional Western blotting. Upon the withdrawal of culture medium and washing in ice-cold phosphate buffered saline (two $75 \mathrm{~cm}^{2}$ flasks per group), cells were lysed with RIPA buffer (89901, Thermo Scientific), and total protein concentration was measured using Pierce BCA Protein Assay Kit (23227, Thermo Scientific). Protein samples (15 $\mu \mathrm{g}$ per sample) were mixed with NuPAGE LDS Sample Buffer (NP0008, Invitrogen) and NuPAGE Sample Reducing Agent (NP0004, Invitrogen), denatured at 99 ${ }^{\circ} \mathrm{C}$ during $5 \mathrm{~min}$, loaded into 10-well NuPAGE 4-12\% Bis-Tris Protein Gels of $1.5 \mathrm{~mm}$ thickness (NP0335BOX, Invitrogen) and separated in NuPAGE MES SDS Running Buffer (NP000202, Invitrogen) containing NuPAGE Antioxidant (NP0005, Invitrogen) at $150 \mathrm{~V}$ for $1.5 \mathrm{~h}$. A 1:1 mixture of Novex Sharp Pre-stained Protein Standard (LC5800, Invitrogen) mixed with MagicMark XP Western Protein Standard (LC5602, Invitrogen) was utilized as a molecular-weight size marker. Dry protein transfer was conducted during a standard 7-minute protocol employing polyvinylidene fluoride iBlot 2 Transfer Stacks (IB24001, Invitrogen) and iBlot 2 Gel Transfer Device (Invitrogen). Membranes were then incubated in iBind Flex blocking solution (SLF2020 $\times 4$, Invitrogen) for $2 \mathrm{~h}$ and then processed in iBind Western Device (Invitrogen) overnight with recommended primary and secondary antibody dilutions (iBind Flex solution). The following primary antibodies was used: mouse anti-CD31 (ab9498, Abcam, 1:1000), rabbit anti-VE-cadherin (36-1900, Invitrogen, 1:100), rabbit anti-vWF (ab6994, Abcam, 1:500), rabbit anti-VEGFR2/KDR (ab39256, Abcam, 1:1000), rabbit anti-CD34 (ab81289, Abcam, 1:1000), rabbit anti-neuropilin 1 (ab81321, Abcam, 1:1000), rabbit anti-HEY2 (ab221931, Abcam, 1:200), mouse anti-NR2F2/COUP-TFII (ab41859, Abcam, 1:200), rabbit anti-LYVE1 (ab14917, Abcam, 1:200), rabbit anti-VEGFR3 (ab27278, Abcam, 1:100), rabbit anti-Snail + Slug (ab180714, Abcam, 1:500), mouse anti-N-cadherin (MA5-15633, Invitrogen, 1:500), and goat anti- $\beta$-tubulin (loading control, ab21057, Abcam, 1:1000). Horseradish peroxidase-conjugated goat anti-mouse (AP130P, Sigma-Aldrich, 1:1000), goat anti-rabbit (7074S, Cell Signaling Technology, 1:200), or donkey anti-goat (ab205723, Abcam, 1:400) were used as secondary antibodies. Chemiluminescence detection was carried out by incubation of the membrane in SuperSignal West Pico PLUS Chemiluminescent Substrate (34580, Thermo Scientific) for 1 min followed by 12-minute exposure in C-DiGit Blot Scanner (LI-COR Biosciences). Dot blotting against 55 angiogenesis-related proteins was performed using Proteome Profiler Human Angiogenesis Array Kit (ARY007, R\&D Systems, Minneapolis, MN, USA) according to the manufacturer's instructions (275 $\mu \mathrm{g}$ of cell lysate per sample was loaded onto the membrane). 


\subsection{Statistical Analysis}

Statistical analysis was performed using GraphPad Prism 8 (GraphPad Software, San Diego, CA, USA). Data were represented by the median, 25th and 75th percentiles, and range. Groups were compared by Mann-Whitney $\mathrm{U}$ test. $P$ values $\leq 0.05$ were regarded as statistically significant.

Statistically significant DEGs were defined as those with fold change $\geq 2$ and false discovery rate (FDR) $p$-value $<0.05$. In Gene Ontology (GO) functional enrichment analysis (CLC GW 12.0), we considered only statistically significant (FDR $p$-value $<0.05$ ) categories with the ratio of DEGs to total genes exceeding $0.5(50 \%)$.

\section{Results}

\subsection{Differentiation of ECFC from PBMC}

To differentiate ECFC from PBMC, we applied the protocol established by Kolbe et al. [23], including culture of PBMC in EGM-2MV medium in collagen-coated flasks with the further reseeding of the intermediate cell population into fibronectin-coated flasks and finally into the standard culture dishes. Both flow cytometry (Figure 1A, Table 1) and confocal microscopy (Figure 1B) confirmed endothelial specification in terms of CD31, vWF, KDR, and CD146 positive staining and CD45-negative staining. ECFC formed capillary-like tubes in Matrigel (Figure 1C) with the characteristics similar to HUVEC (Figure 1D), internalized acLDL with the concurrent UEA binding (Figure 2A), and exhibited a remarkable proliferation capability superior to HUVEC (Figure 2B-D), thereby fulfilling requirements for endothelial identity.

Table 1. Median fluorescence intensities registered for different antigens and cell lines during flow cytometry analysis.

\begin{tabular}{ccccccc}
\hline Antigen & \multicolumn{2}{c}{ ECFC } & \multicolumn{2}{c}{ HUVEC } & \multicolumn{2}{c}{ SAT-SVF } \\
\hline & Isotype & Sample & Isotype & Sample & Isotype & Sample \\
CD34 & 3080 & 7155 & 1579 & 5220 & 3406 & 9438 \\
CD309 & 4257 & 15,063 & 2272 & 13,974 & 1640 & 2155 \\
CD146 & 5080 & $1,262,908$ & 210 & 417,263 & 256 & 445 \\
CD133 & 24,425 & 24,390 & 2083 & 1891 & 0 & 0 \\
CD45 & 4128 & 4221 & 2353 & 2266 & 25,102 & 24,684 \\
CD31 & 26,148 & $2,151,501$ & 65 & 44,055 & 362 & 1213 \\
vWF & 4243 & 203,182 & 668 & 127,268 & 156,915 & 167,111 \\
CD73 & 0 & 0 & 0 & 0 & 180 & 8235 \\
CD90 & 4883 & 4915 & 4531 & 4598 & 1133 & 2934 \\
\hline
\end{tabular}

\subsection{ECFC and SAT-SVF Have Remarkable Differences in Global Gene Expression Profile}

For the comprehensive and unbiased evaluation of the baseline gene expression profile in ECFC as compared with HCAEC and HUVEC, we performed RNA-seq of indicated cell cultures, suggesting SAT-SVF as a negative control population according to the immunophenotyping and functional analyses (Figure S3). A total of 2696 DEGs were identified between ECFC and SAT-SVF (Figure S4; Supplementary Datasheet 1); hierarchical clustering by the transcription level of top DEGs revealed a clearly defined aggregation of RNA samples from SAT-SVF and ECFC into separate clusters (Figure S5). These results have been further confirmed by a principal component analysis (Figure S6). 
A
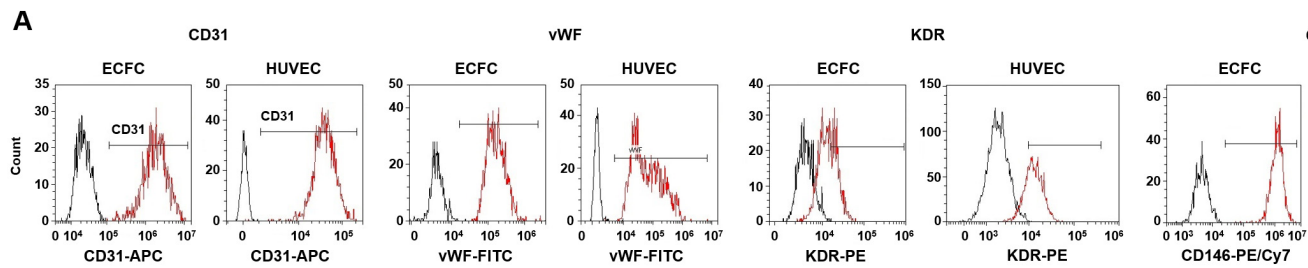

CD146
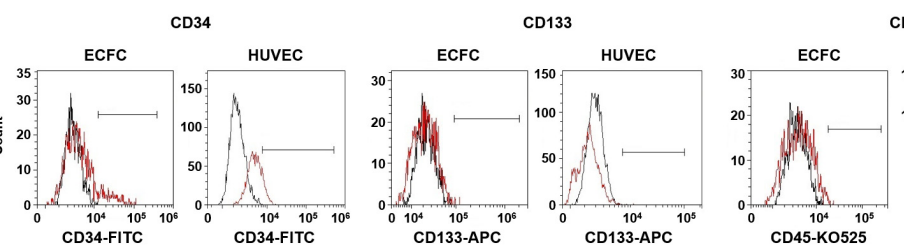

CD45

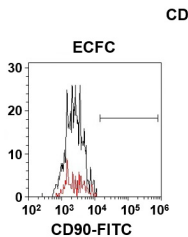

CD90
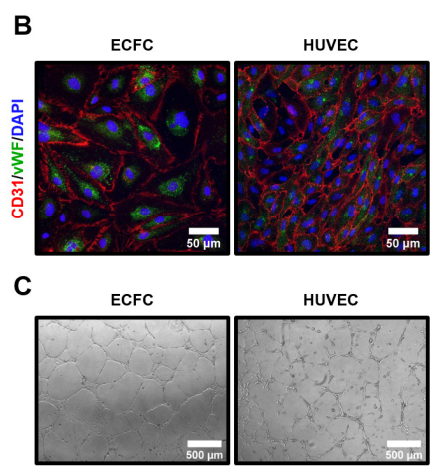

D
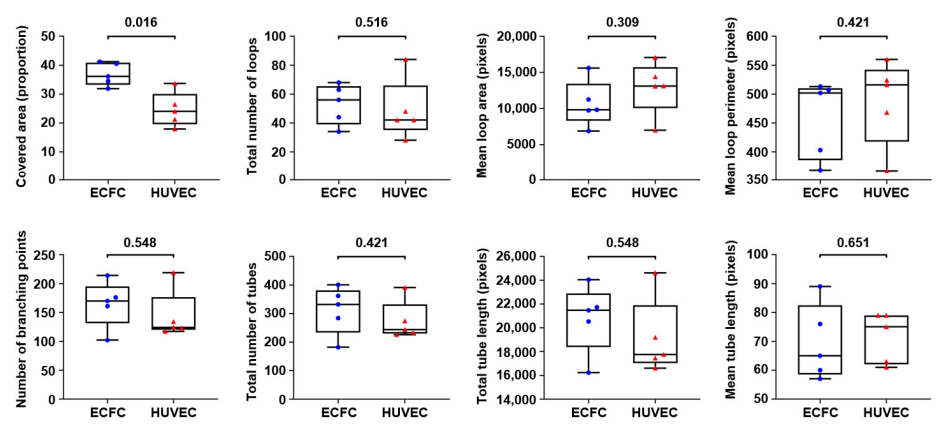
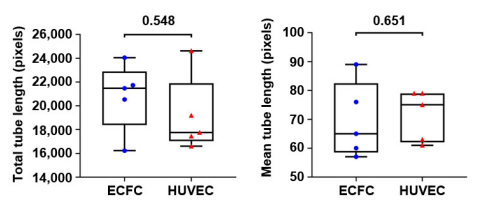

Figure 1. Peripheral blood mononuclear cells (PBMC)-derived endothelial colony-forming cells (ECFC) have characteristic endothelial immunophenotype and considerable tube-forming activity in Matrigel. (A) Flow cytometry immunophenotyping (representative graphs) found both ECFC and human umbilical vein endothelial cells (HUVEC) having CD31+vWF+KDR+CD146+CD34-CD133-CD45-CD90expression signature suggestive of endothelial identity. Black color defines isotype control while red color is for specific staining; (B) Confocal microscopy (representative images) corroborates flow cytometry results demonstrating CD31-positive (red color, cell surface receptor) and vWF-positive (green color, Weibel-Palade bodies inside the cytosol) ECFC and HUVEC. Nuclear counterstaining was performed using 4',6-diamidino-2-phenylindole (DAPI, blue color); (C) Phase contrast microscopy (representative images). Both ECFC and HUVEC have pronounced capability to assemble capillary-like tubes in Matrigel; (D) Semi-quantitative analysis of the tube-forming activity from the experiment in C. Each dot represents a representative image from one well of the culture plate ( $n=5$ wells per group). Whiskers indicate range, box bounds indicate 25 th and 75 th percentiles, center lines indicate median. $P$ values are provided above the graphs in a numerical manner according to Mann-Whitney U test.

\subsection{ECFC Demonstrate High Similarity to Resident Vascular EC yet Represent a Transitional Cell Population}

We next compared the baseline gene expression profile of ECFC to HCAEC (Supplementary Datasheet 2) and HUVEC (Supplementary Datasheet 3). Hierarchical clustering demonstrated certain transcriptomic signatures for both of these comparisons (Figure 3). When ECFC were compared with HCAEC, gene set enrichment analysis revealed significant differences in gene expression profile exclusively in vascular endothelial growth factor-activated receptor activity (Table S2). However, it neither detected other statistically significant differences between ECFC and HCAEC GO categories nor observed any differences between ECFC and HUVEC. With the aim to pinpoint DEGs between ECFC, HCAEC, and HUVEC, we manually annotated them regarding their role in endothelial biology. 
A

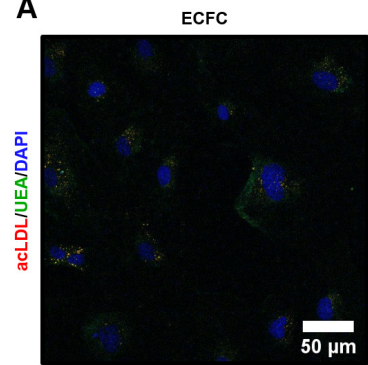

B

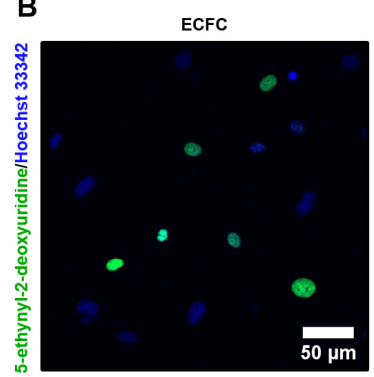

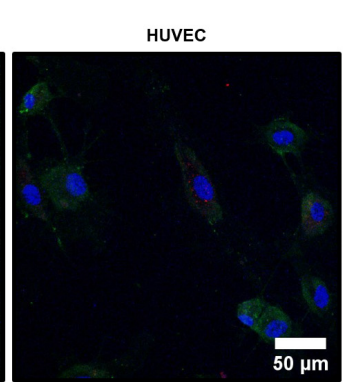

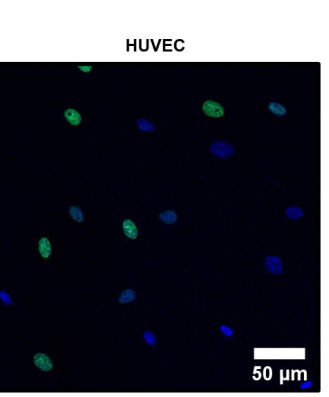

C

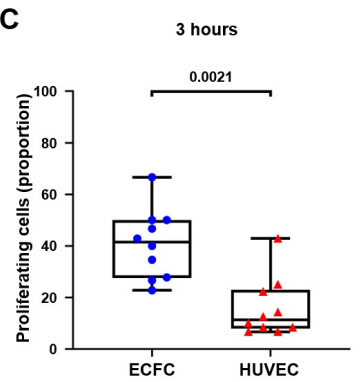

D

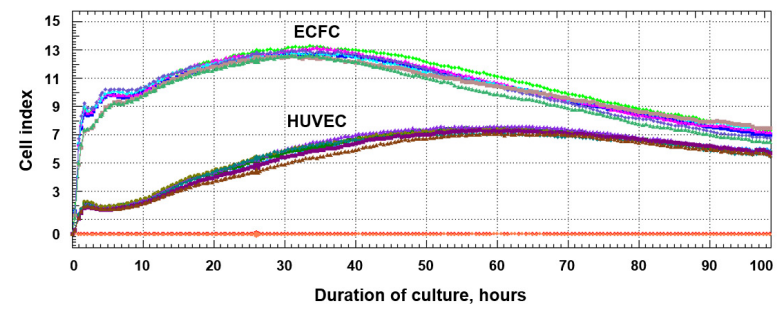

Figure 2. PBMC-derived ECFC internalize acetylated low-density lipoprotein cholesterol (acLDL), bind Ulex Europaeus agglutinin 1 (UEA), and exhibit high proliferation capacity. (A) Confocal microscopy (representative images) shows uptake of acLDL (red color inside the cytosol) and binding of UEA (green color around the cell membrane) by ECFC and HUVEC. Nuclear counterstaining was performed using DAPI (blue color); (B) A major proportion of ECFC and HUVEC are actively dividing as shown by 5-ethynyl-2-deoxyuridine incorporation into their nuclei (Hoechst 33342, blue). Representative images; (C) Semi-quantitative analysis of the proliferative activity from the experiment in B. Each dot represents one representative image from one well of the culture plate ( $n=10$ wells per group). Whiskers indicate range, box bounds indicate 25 th and 75 th percentiles, center lines indicate median. $P$ values are provided above the graphs in a numerical manner according to Mann-Whitney U test; (D) Electrical impedance monitoring in ECFC and HUVEC cultures confirms the results from confocal microscopy. Proliferation capability was defined as cell index doubling time calculated automatically by the instrument software. Each line represents a result collected from one well of the culture plate $(n=7$ per group). Red lines at the bottom indicate impedance of the blank solution (cell-free culture medium).

According to such annotation, when compared with HCAEC, ECFC had a higher expression of pan-endothelial markers KDR (2.2-fold) and VWF (3.9-fold), endothelial progenitor cell marker CD34 (23.9-fold), venous endothelial specification marker NRP2 (7.9-fold), lymphatic endothelial lineage markers FLT4 (11.6-fold) and LYVE1 (45.7-fold), subunits of collagen type IV composing endothelial basement membrane (COL4A1 and COL4A2, 2.5 and 3-fold, respectively), and subunits of collagen type I, a major component of the extracellular matrix (COL1A1 and COL1A2,926-fold and 43.5-fold, respectively). In contrast, HCAEC had higher expression of the gene encoding key Notch pathway transcription factor HEY2 (523-fold), NOS3 gene (4.9-fold), and FLT1 gene (encodes VEGFR1, 3.9-fold). Comparison in terms of manually annotated categories found that HCAEC overexpressed genes encoding proteins responsible for vascular development (5 versus 0 in ECFC) while ECFC overexpressed genes providing ECM production/remodeling (13 versus 7 in HCAEC) and endothelial integrity (15 versus 8 in HCAEC); other categories did not show any notable differences (Figure 4A). 
A

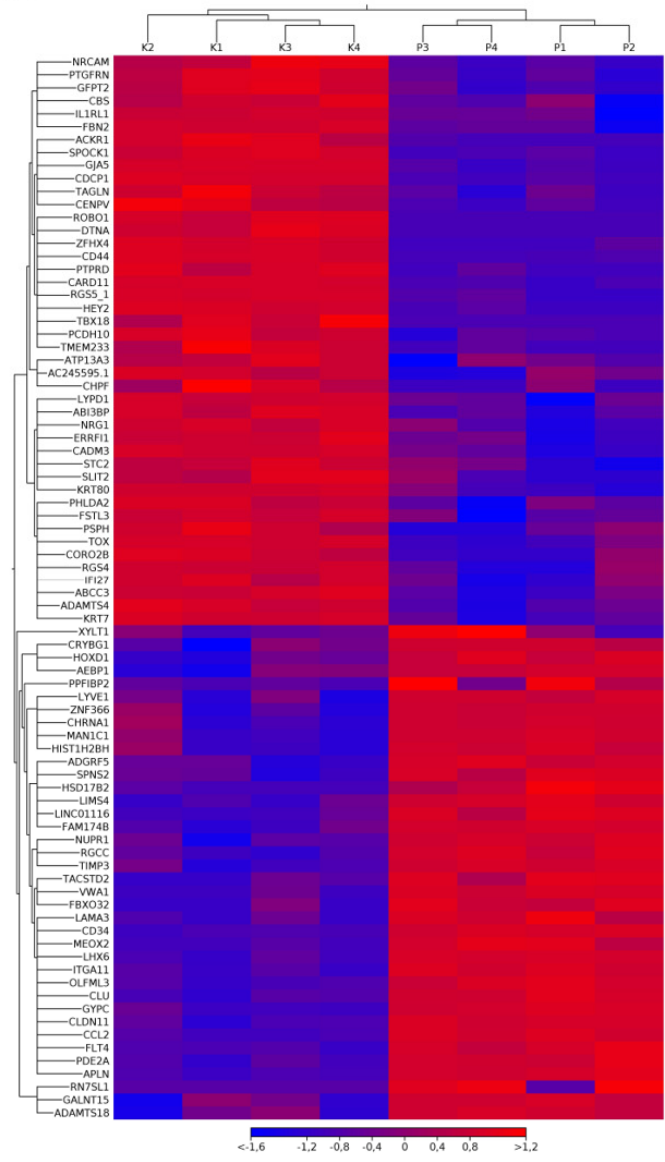

B

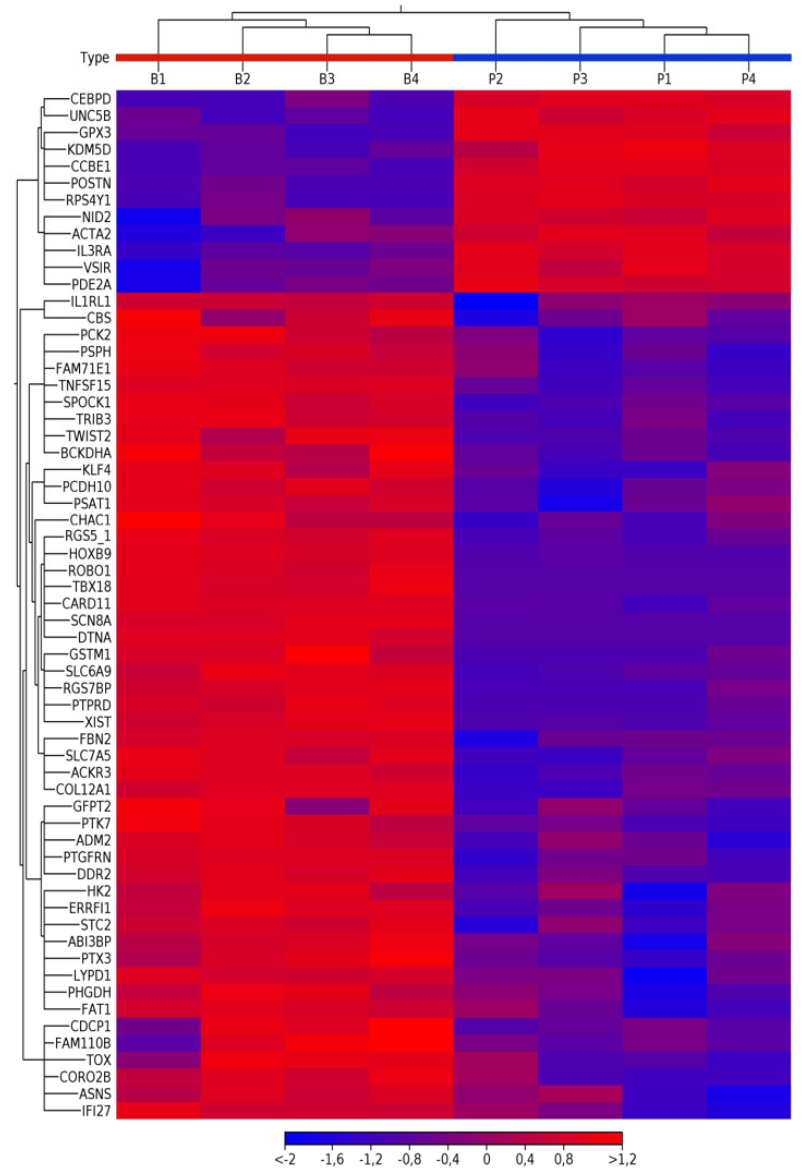

Figure 3. Heatmaps of differentially expressed genes between (A) human coronary artery endothelial cells (HCAEC) vs. ECFC (only those having fold change $0.1(-10)$ or 10, respectively, and FDR $p$-value < 0.05 are represented in the heatmap); (B) HUVEC vs. ECFC (only those having fold change $0.2(-5)$ or 5 , respectively, and FDR $p$-value $<0.05$ are represented in the heatmap). Top panel: dendrogram showing the results of hierarchical sample clustering. Left panel: Dendrogram showing the results of hierarchical differentially expressed genes (DEGs) clustering. DEGs labeling based on Ensembl annotation.

In comparison with HUVEC, ECFC had higher expression of NOTCH4 gene (3.1-fold), Notch ligand DLL2 (4.1-fold), LYVE1 (18.7-fold), and COL1A1 (914-fold), while HUVEC had higher expressions of the key endothelial mechanotransduction transcription factor KLF4 (45-fold), mesenchymal marker CDH2 (encodes N-cadherin, 7.6-fold), and VEGFA (5-fold). Comparison of ECFC to HUVEC regarding manually annotated categories demonstrated that HUVEC overexpressed genes encoding proteins stimulating migration of EC (4 versus 0 in ECFC) and angiogenesis (19 versus 6 in ECFC); other categories displayed no appreciable differences (Figure 4B).

As compared with other cells of endothelial identity, ECFC were characterized by a higher baseline expression of the genes responsible for basement membrane composition (COL4A1 and COL4A2 encoding collagen type IV) and extracellular matrix (COL1A1 and COL1A2 encoding collagen type I) and expressed markers of all three endothelial lineages (i.e. arterial, venous, and lymphatic specification), thereby showing a transitional transcriptomic signature. Functional enrichment of the GO-defined gene networks responsible for endothelial homeostasis and angiogenesis (Supplementary Datasheet 4) demonstrated a higher similarity to HUVEC (32 DEGs out of 653 included into this subanalysis) than to HCAEC (87/653), albeit HCAEC and HUVEC differed more significantly (112/653) than each of these populations compared with ECFC (Supplementary Datasheet 5 and Figure 5). In addition to functional enrichment analysis, a Venn diagram (Figure 6) and principal component analysis (Figure 7), 
which included all DEGs, also indicated that the gene expression profile of ECFC is closer to HUVEC (261 DEGs) than to HCAEC (470 DEGs); nevertheless, differences between HCAEC and HUVEC were not more pronounced when all DEGs were included into the analysis (420 DEGs). Analysis of manually annotated categories found comparable differences in both number of differentially expressed categories and DEGs within them between ECFC, HCAEC, and HUVEC (two categories per ECFC-HCAEC and ECFC-HUVEC comparisons including 28 and 29 DEGs, respectively). Manual screening of pluripotency markers documented only scarce differences between ECFC, HCAEC, and HUVEC, but the differences between HCAEC and HUVEC (7 DEGs) were higher than in pairwise comparison of ECFC with either HCAEC (5 DEGs) or HUVEC (4 DEGs) (Figure 8). Taken together, these results suggested intermediate specification of ECFC in relation to HCAEC and HUVEC.

A

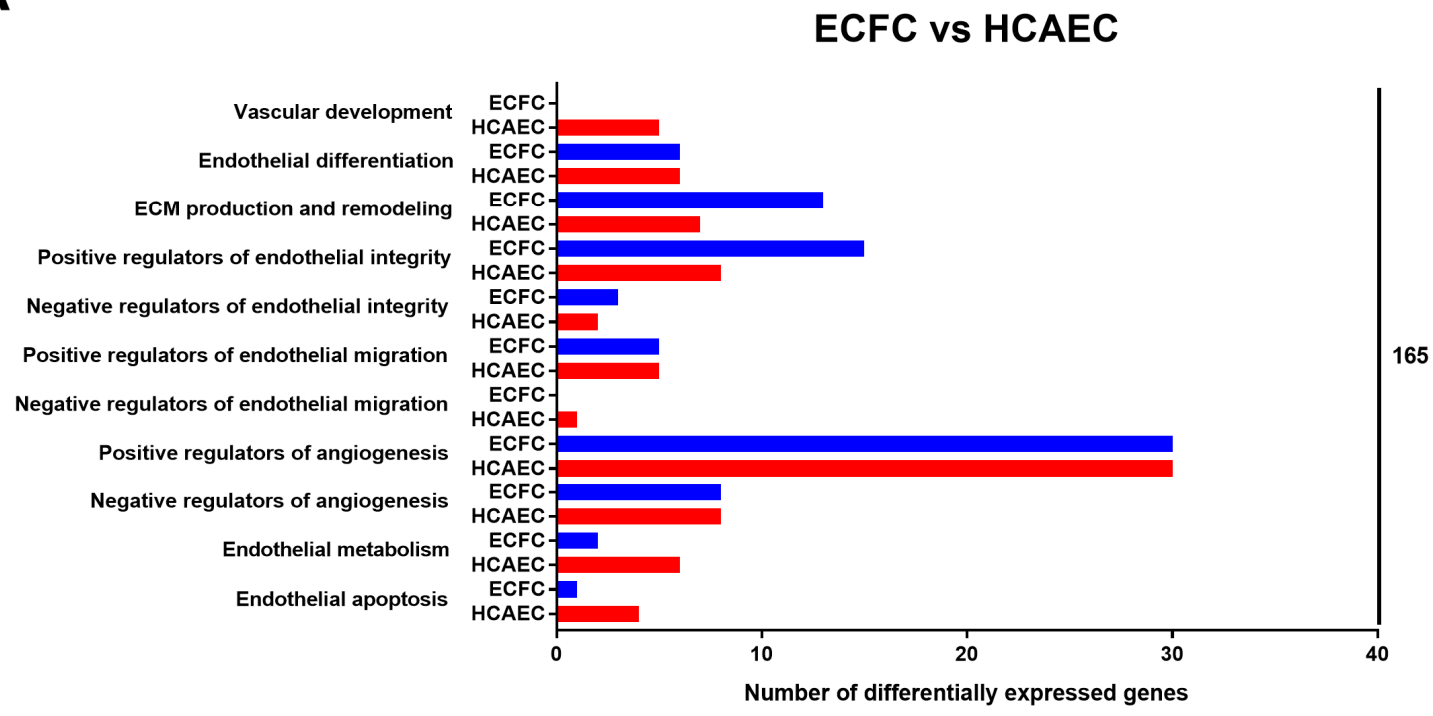

B

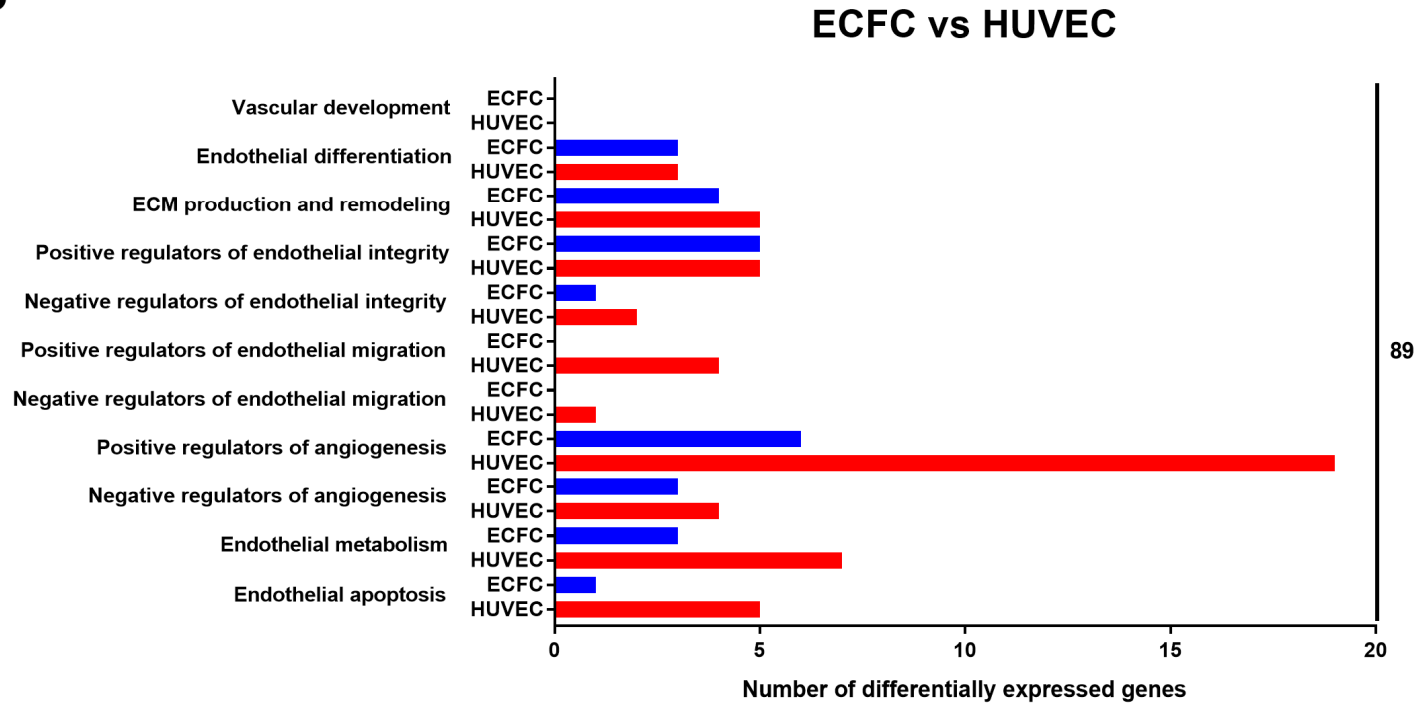

Figure 4. Pairwise comparison of (A) ECFC versus HCAEC and (B) ECFC versus HUVEC according to the manual annotation of DEGs (only endothelial biology-related categories shown). Total number of DEGs within the indicated categories is provided as the count at the right side of the graph. 
ECFC vs. HUVEC ECFC vs. HCAEC HCAEC vs. HUVEC

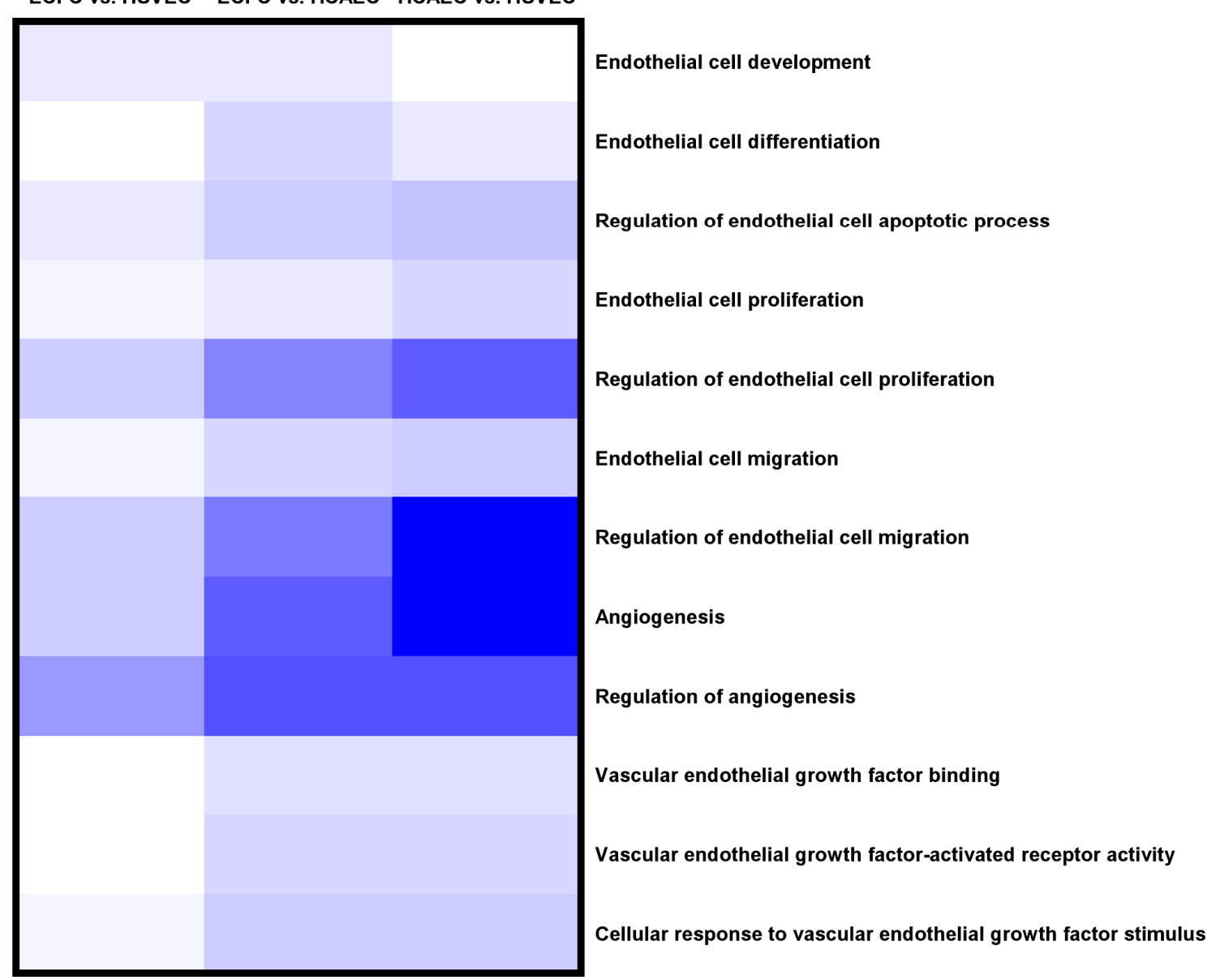

Total number of DEGs included into the heatmap

Number of DEGs in GO category

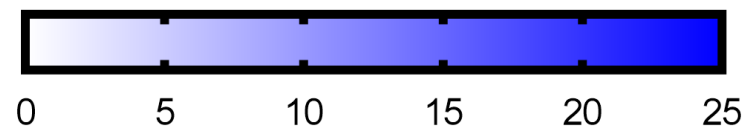

Figure 5. Heatmap representing the genes related to the regulation of endothelial homeostasis and angiogenesis and differentially expressed between ECFC, HCAEC, and HUVEC according to the GO functional enrichment analysis. Top panel: pairwise comparisons between the EC populations. Right panel: GO terms manually included in the heatmap. Bottom panel: a color mapping range. 


\section{ECFC vs HUVEC (261 differentially expressed genes)}

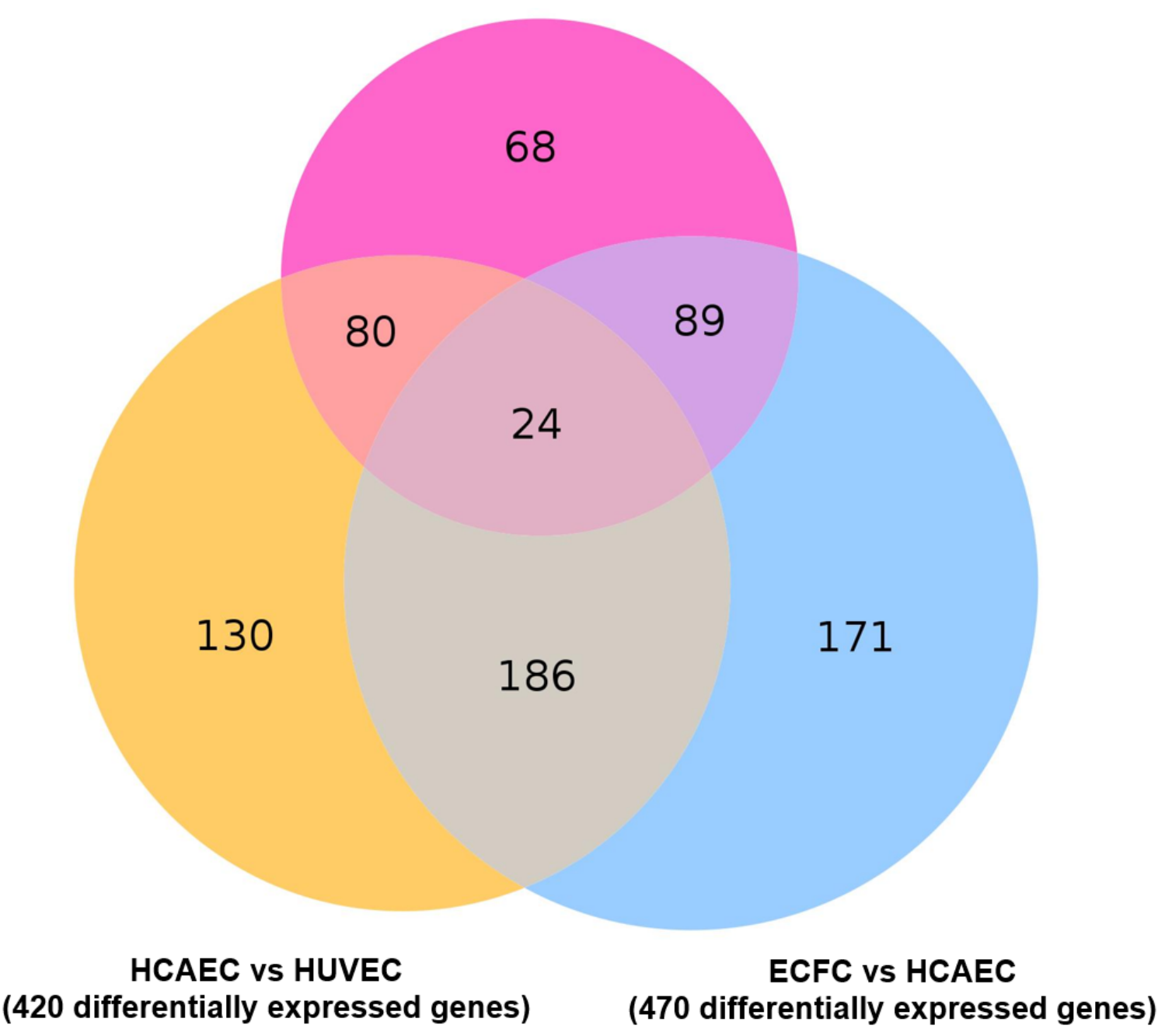

Figure 6. Venn diagram for the genes differentially expressed in ECFC, HUVEC and HCAEC.

3.4. Proteomic Profiling Validates Transcriptome-Wide Comparison Confirming Intermediate Endothelial Hierarchy of ECFC

To verify the key results obtained by RNA-seq, we measured the relative levels of endothelial lineage markers and angiogenic molecules across SAT-SVF, ECFC, HCAEC, and HUVEC utilizing different Western blotting modalities. Indeed, abundant expression of angiopoietin 2 (Ang-2) and endothelin-1 (ET-1) has been detected in the lysate of ECFC but not SAT-SVF in accordance with the fold change values acquired from RNA-seq (1600 and 817, respectively); however, the reverse was the case for tissue inhibitor of metalloproteinases 1 (TIMP-1, fold change -10, Figure S7).

Similar to RNA-seq, proteomic profiling did not find major differences across the EC, yet ECFC generally had either the highest or lowest expression of angiogenic molecules in head-to-head comparison with HCAEC and HUVEC (Figure 9A). Analysis of pan-endothelial markers verified the endothelial phenotype of ECFC which had high levels of CD31, VE-cadherin, VEGFR2/KDR, CD34, and neuropilin-1 (NRP1) (Figure 9B). Blotting against endothelial lineage and endothelial-to-mesenchymal transition markers testified to the transitional phenotype of ECFC in terms of intermediate HEY2, LYVE1, VEGFR3, Snail, and Slug content when compared to HCAEC and HUVEC, which overexpressed HEY2 and COUP-TFII, respectively (Figure 9B). 


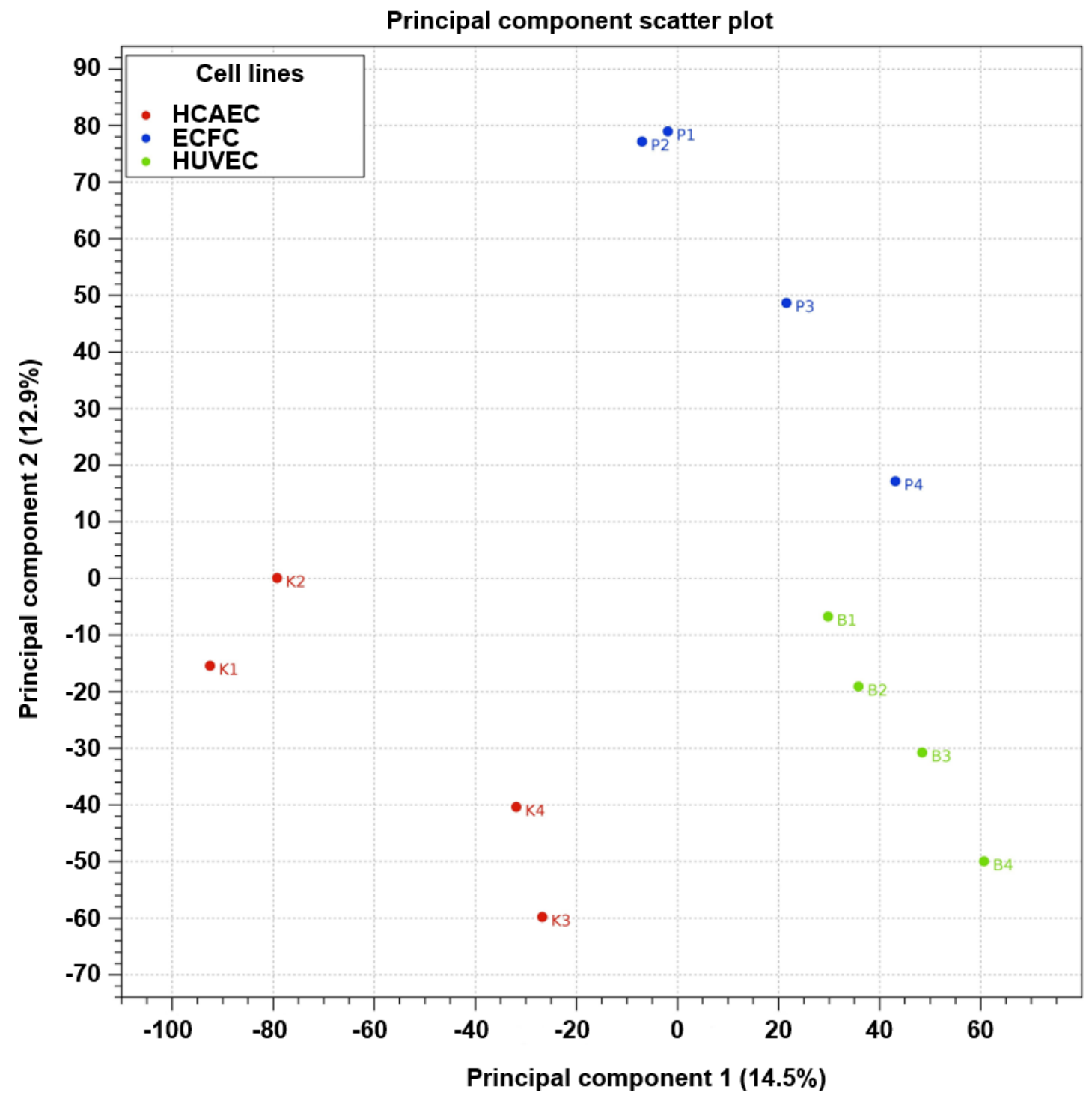

Figure 7. 2D sample clustering based on a principal component analysis (PCA) of differentially expressed genes (DEGs). The red, blue, and green dots indicate HCAEC, ECFC, and HUVEC samples, respectively. Principal components are defined along the $\mathrm{X}$ and $\mathrm{Y}$ axes as component 1 and component 2, respectively; the proportion of explained data variance is indicated in brackets for each principal component.

Differentially expressed pluripotency markers
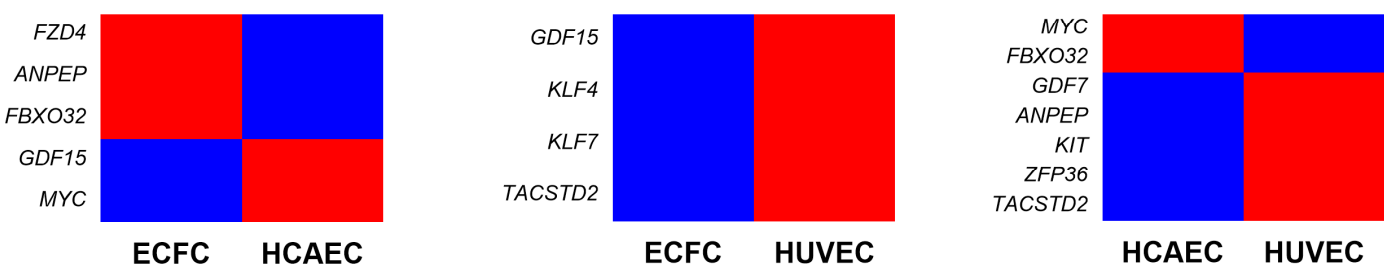

Figure 8. Heatmap showing the differential expression of pluripotency markers across ECFC, HCAEC, and HUVEC. Upregulated genes are colored red whereas downregulated genes are colored blue. 
A

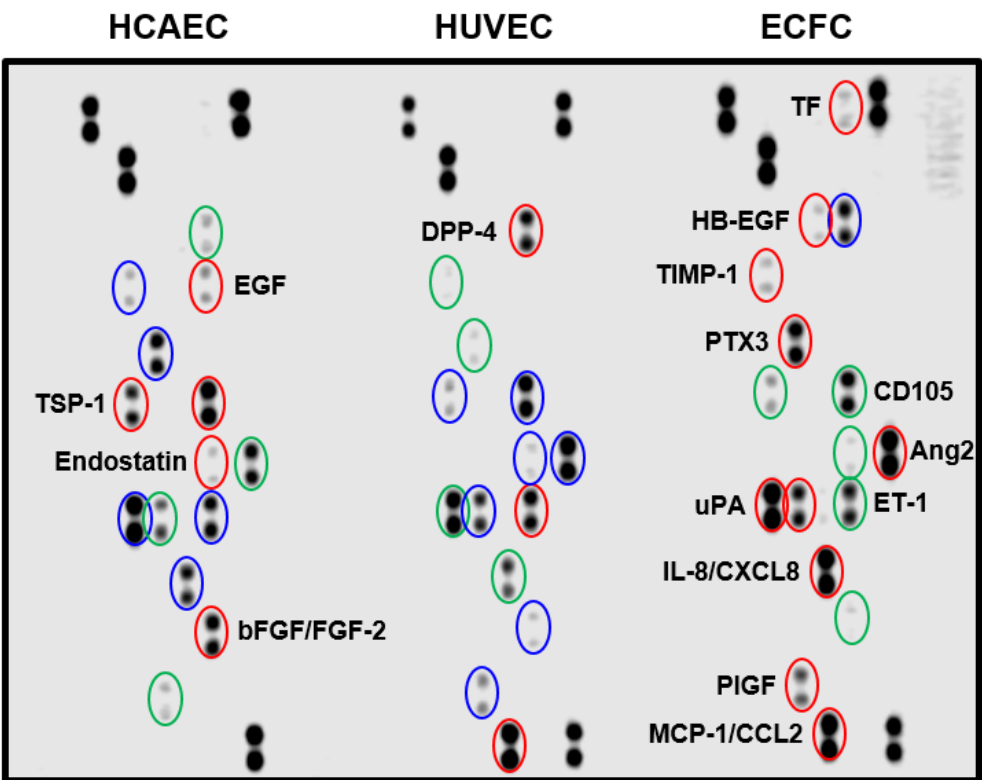

$\bigcirc$ High expression $\bigcirc$ Intermediate expression

Low expression

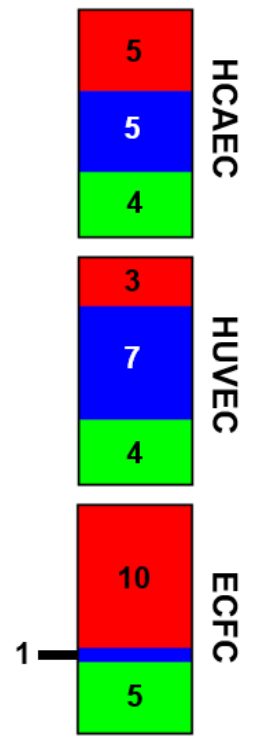

B

Pan-endothelial markers

ECFC HCAEC HUVEC SAT-SVF

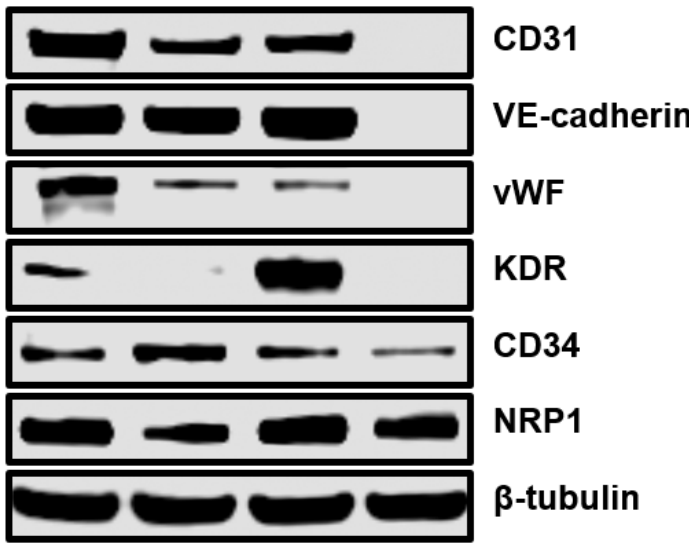

Endothelial lineage and EndoMT markers

ECFC HCAEC HUVEC SAT-SVF

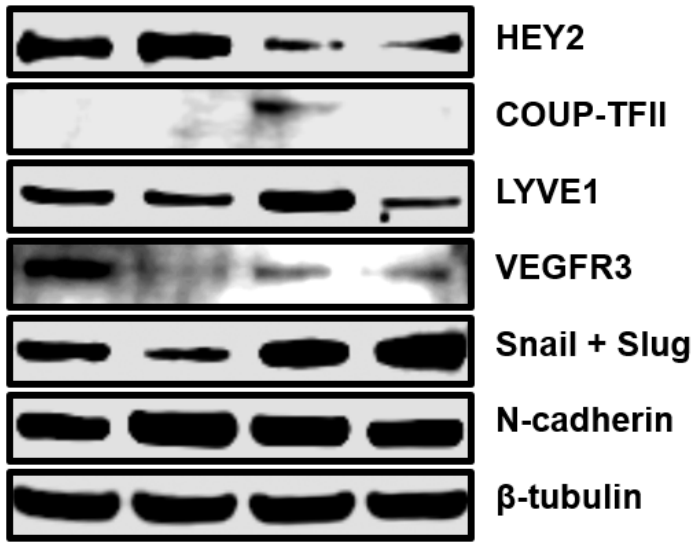

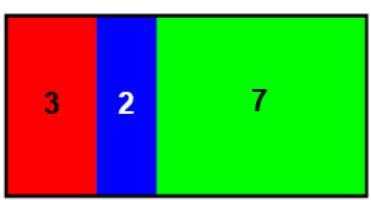

HCAEC

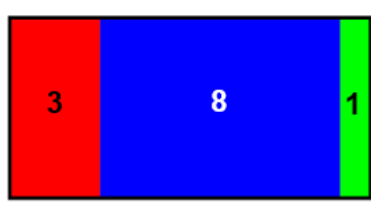

ECFC

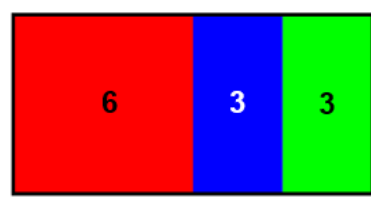

HUVEC

Figure 9. Proteomic profiling for ECFC, HCAEC and HUVEC. (A) Dot blot profiling for 55 angiogenesis-related proteins confirms high similarity between ECFC and mature vascular EC yet also indicates minor differences between these cell lines and generally validating RNA-seq results. Count to the right represents a quantitation of measured angiogenesis-related proteins in terms of their relative expression in HCAEC, HUVEC, and ECFC; (B) Western blotting for endothelial phenotype-related markers verifies endothelial identity of PBMC-derived ECFC and demonstrates an intermediate phenotype of PBMC-derived ECFC as compared to HCAEC and HUVEC in terms of HEY2, LYVE1, VEGFR3, Snail and Slug expression. $\beta$-tubulin was probed as a loading control. Count to the bottom represents a quantitation of measured endothelial phenotype-related markers in terms of their relative expression in HCAEC, HUVEC, and ECFC. 


\section{Discussion}

ECFC represent a cell population that can be differentiated from peripheral blood $[12,13,25]$, umbilical cord blood [25-28], placenta [29-31], bone marrow [32], white adipose tissue [33], peripheral lung tissue [34] and induced pluripotent or embryonic stem cells [35], and is characterized by an endothelial immunophenotype (CD31+vWF+KDR+CD146+CD45-), intracellular CD133 expression, acLDL uptake and UEA binding, considerable tube-forming capacity and high proliferation capability $[16,25,28]$. The combination of endothelial identity with high proliferative and neovascularization potential suggests ECFC as an efficient tool for regenerative medicine applications including pre-endothelialization [36] and pre-vascularization [27,37-39] of tissue-engineered constructs. However, usefulness of ECFC for indicated tasks depends to a large extent on their similarity to resident EC, such as HCAEC, that are particularly important for pre-seeding of small-diameter vascular grafts, or HUVEC which represent the far most frequently utilized endothelial cell line in biomaterial research [17]. As phenotypic features are tightly regulated by the gene expression networks, we sought to investigate the transcriptome profile of ECFC, HCAEC, and HUVEC in comparison with conceivably different SAT-SVF.

We found major differences in gene networks responsible for VEGF signaling, proliferation, migration, organization of intercellular junctions, and neovascularization between ECFC and SAT-SVF. Further, expression of endothelial signature genes (PECAM1, KDR, CDH5, FLT1, VWF, TIE1, CD34, NOTCH1, NOTCH4, CXCR4, FLI1, MYO5C, ANGPT2), basement membrane genes (COL4A1/COL4A2), and NOS3 gene encoding endothelial nitric oxide synthase, a crucial enzyme for maintenance of endothelial homeostasis, was significantly increased in ECFC. Taken together with a pronounced tube-forming activity of ECFC in a 3D culture model (Matrigel) and distinct immunophenotype, these findings may indicate a higher capability of CD31+vWF+KDR+CD146+ ECFC to assemble blood vessels as compared with CD31-vWF-KDR-CD146-SAT-SVF. In contrast to our results, a similar study identified minor differences between immunophenotype, tube-forming capacity, and global gene expression profiles (223 DEGs compared with 2696 in our study) of ECFC and SAT-SVF-derived EC, possibly due to the additional enrichment of SVF by removal of CD44+ cells [40].

Expectedly, ECFC were substantially more similar to HCAEC (470 DEGs) and HUVEC (261 DEG) than to SAT-SVF (2696 DEGs). The extent of heterogeneity between HCAEC and HUVEC (420 DEGs) generally did not exceed that in ECFC-HCAEC and ECFC-HUVEC comparisons, suggesting minor yet significant differences between these endothelial cell populations. Gene set enrichment analysis and screening of pluripotency markers did not uncover notable differences; however, manual annotation of DEGs indicated that ECFC overexpress markers of all three endothelial differentiation lineages (KDR, VWF, CD34, NRP2, FLT4, and LYVE1 in comparison with HCAEC; NOTCH4, DLL2, and LYVE1 when compared with HUVEC).

Such an expression pattern assumes that ECFC are able to be differentiated into arterial, venous, or lymphatic endothelium, and might be closer to the latter lineage than HCAEC or HUVEC because of higher LYVE1 expression. Moreover, ECFC have higher expression of venous endothelial marker NRP2 than HCAEC, but also overexpress arterial differentiation marker NOTCH4 as compared with HUVEC, suggestive of their intermediate specification between arterial and venous EC. This is corroborated by increased COL1A1 expression by ECFC in comparison with HCAEC and HUVEC and augmented COL1A2, COL4A1, and COL4A2 expression in ECFC compared with HCAEC, testifying to the upregulated production of extracellular matrix, including major components of the basement membrane. Importantly, proteomic profiling confirmed the results of RNA-seq, as levels of endothelial markers and angiogenesis molecules in ECFC were also intermediate between HCAEC and HUVEC. We therefore suggest PBMC-derived ECFC as a transitional cell population in the endothelial hierarchy (Figure 10). 
ECFC as a transitional cell population in the endothelial hierarchy

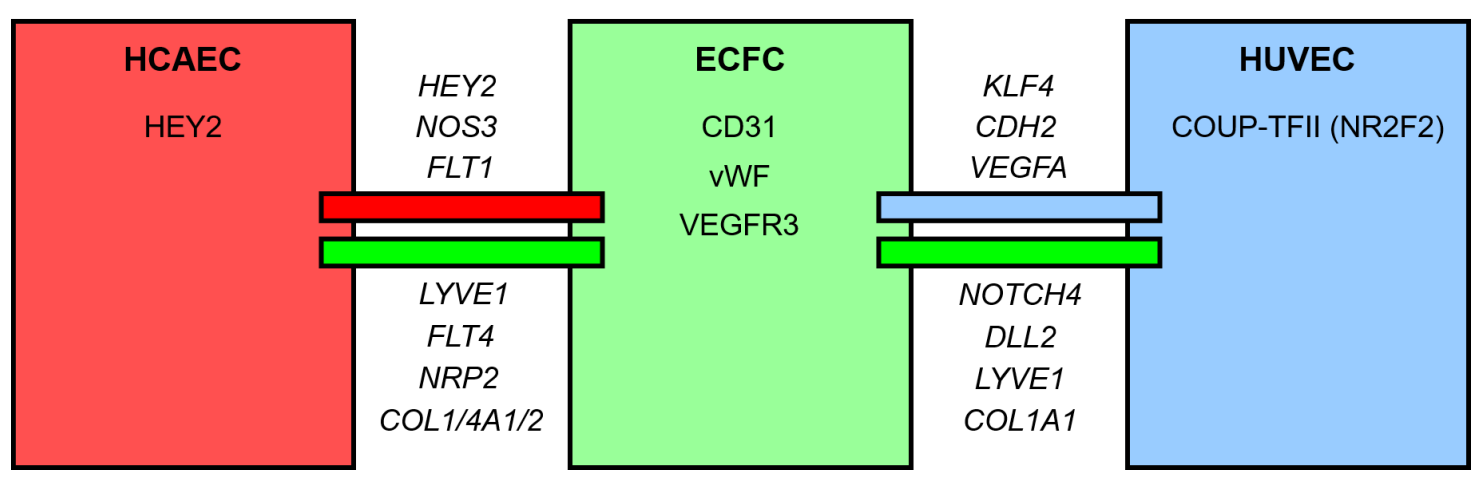

Figure 10. Graphical summary of the results. We suggest ECFC as a transitional cell population in the endothelial hierarchy overexpressing extracellular matrix/basement membrane components, lymphatic endothelial markers, and also venous endothelial markers as compared with mature arterial ECs and arterial endothelial markers in comparison with mature venous ECs. Changes at the protein level are within the boxes whereas changes at the transcriptomic level are above or below the lines (italicized gene names). Genes above the red and blue lines are upregulated in HCAEC and HUVEC, respectively, as compared with ECFC. Genes below the green lines are upregulated in ECFC in comparison with HCAEC (left green line) and HUVEC (right green line), respectively.

Previous reports demonstrated that the global gene expression profile of ECFC largely depends on the source of their isolation and it varies between adult peripheral and cord blood [41], between the cultures grown in complete and serum-free medium $(\approx 1100$ DEGs) [42], and between blood collected from the patients with breast or renal cancer and healthy donors (342 and 71 DEGs, respectively) [43]. Intriguingly, ECFC isolated from breast and renal cancer patients shared a common 35-gene signature [43]. Cord-blood-derived ECFC from preterm neonates had $>700$ DEGs in comparison with term newborns [44] while placental- and cord-blood-derived ECFC differed to a negligible extent (33 DEGs with fold change cutoff of 1.5) [30].

Co-culture of ECFC with mesenchymal stromal cells significantly alters their gene expression program $(\approx 1700 \mathrm{DEGs})$ triggering endothelial-to-mesenchymal transition, potentially through elevated expression of SNAI1 and SNAI2 genes [45] encoding, respectively, transcription factors Snail and Slug, master regulators of this process [46-48]. Interestingly, in our study, SNAI1 was upregulated whilst SNAI2 was downregulated in ECFC compared with SAT-SVF, probably reflecting different stages of endothelial differentiation. Additionally, separate colonies within the single ECFC isolate may have distinct immunophenotype and transcriptomic signatures, e.g., arterial or lymphatic, and the extent of such heterogeneity ( $\approx 2250$ DEGs) exceeded those obtained in our experiments (200-500 DEGs) and when blood and lymphatic ECFC were compared with primary mature dermal microvascular EC $(\approx$ 1900 DEGs) [49]. A recent study proposed cytokine-like 1 (CYTL1), a protein promoting angiogenic sprouting, as a reliable marker of peripheral- and cord-blood-derived ECFC but not HUVEC or human saphenous vein EC being consistently $>10$-fold overexpressed in ECFC [50]. This was partially replicated in our analysis as ECFC had 2.5- and 830-fold higher CYTL1 expression than HUVEC and SAT-SVF, respectively.

To the best of our knowledge, this study is the first attempt to conduct a transcriptome-wide comparison of ECFC to mature vascular EC populations such as HCAEC or HUVEC. We found that the global gene expression profile of ECFC was close to HCAEC or HUVEC, yet ECFC had transcriptomic signatures such as overexpressed common markers of all endothelial lineages and upregulated extracellular matrix genes. Transcriptome of ECFC drastically differed from SAT-SVF, attesting endothelial commitment of ECFC. 


\section{Conclusions}

The gene expression profile and behavior of PBMC-derived ECFC show a sufficient extent of similarity to vascular EC for testing them in pre-endothelialization of bioartificial vascular grafts, whereas in terms of developmental biology they significantly differ from HCAEC and HUVEC in the expression of certain gene categories relevant for endothelial biology (endothelial specification markers and synthesis of extracellular matrix/basement membrane components).

Supplementary Materials: The following are available online at http://www.mdpi.com/2073-4409/9/4/876/s1, Figure S1: Total RNA size distribution (Bioanalyzer 2100), Figure S2: DNA library size distribution (Bioanalyzer 2100), Figure S3: SAT-SVF have a distinct immunophenotype and negligible tube formation activity in comparison with ECFC (a) Confocal microscopy (representative images) demonstrates a lipid droplet (Nile Red staining, red color) with a small nucleus at its periphery (DAPI, blue color); (b) Flow cytometry immunophenotyping (representative graphs) defines SAT-SVF as CD31-vWF-KDR-CD146-CD34+CD73+CD45-CD90- population. Black color defines isotype control while green color is for specific staining; (c) Phase contrast microscopy (representative images of two different cultures). ECFC have significant tube forming capacity whereas SAT-SVF generally do not form tube-like structures in Matrigel), Figure S4: Venn diagram for the genes differentially expressed in ECFC, HUVEC, HCAEC, and SAT-SVF, Figure S5: A heatmap of differentially expressed genes between SAT-SVF vs. ECFC (only those having fold change $0.001(-1000)$ or 1000, respectively, and FDR $p$-value $<0.05$ are represented in the heatmap). Top panel: dendrogram showing the results of hierarchical sample clustering. Left panel: Dendrogram showing the results of hierarchical DEGs clustering. DEGs labeling based on Ensembl annotation, Figure S6: 2D sample clustering based on a principal component analysis of differentially expressed genes. The blue, red, green, and purple dots indicate ECFC, HCAEC, HUVEC, and SAT-SVF samples, respectively. Principal components are defined along the $\mathrm{X}$ and $\mathrm{Y}$ axes as component 1 and component 2, respectively; the proportion of explained data variance is indicated in brackets for each principal component, Figure S7: Dot blot profiling for 55 angiogenesis-related proteins shows striking differences between ECFC and SAT-SVF. Table S1: Quality control of RNA, DNA libraries, and RNA-seq results, Table S2: Functional enrichment analysis of differentially expressed genes (DEGs) between ECFC and HCAEC according to the Gene Ontology (GO) categories (only categories with the proportion of DEGs within the GO term to the total number of genes within the GO term $>50 \%$ showed). Supplementary Datasheet 1: Transcriptome-wide comparison of the gene expression profile, SAT-SVF vs. ECFC, Supplementary Datasheet 2: Transcriptome-wide comparison of the gene expression profile, ECFC vs. HCAEC, Supplementary Datasheet 3: Transcriptome-wide comparison of the gene expression profile, ECFC vs. HUVEC, Supplementary Datasheet 4: Pairwise comparison of DEGs within the endothelial biology-related GO terms between ECFC, HCAEC, and HUVEC. Supplementary Datasheet 5: Transcriptome-wide comparison of the gene expression profile, HCAEC vs. HUVEC.

Author Contributions: Conceptualization, A.G.K., M.R.K. and E.A.V.; data curation, A.E.T.; formal analysis, A.G.K., M.R.K. and E.A.V.; funding acquisition, E.A.V.; investigation, A.G.K., A.E.T., V.G.M., D.K.S., L.V.A., M.R.K. and E.A.V.; methodology, A.G.K., A.E.T., V.G.M., D.K.S., L.V.A., M.R.K. and E.A.V.; project administration, E.A.V.; resources, L.V.A. and E.A.V.; software, A.E.T. and M.R.K.; supervision, A.G.K.; validation, A.E.T. and M.R.K.; visualization, A.G.K. and M.R.K.; writing—original draft, A.G.K. and M.R.K.; writing—review \& editing, A.G.K. and M.R.K. All authors have read and agreed to the published version of the manuscript.

Funding: This study was funded by the Russian Science Foundation, grant number 17-75-20004 “Development of personalized tissue-engineered, small-diameter vascular graft in vitro under pulsatile flow conditions". Bioinformatic analysis was supported by Russian State funded budget project AAAA-A17-117020210021-7.

Conflicts of Interest: The authors declare no conflict of interest. The funders had no role in the design of the study; in the collection, analyses, or interpretation of data; in the writing of the manuscript, or in the decision to publish the results.

\section{References}

1. Cuminetti, G.; Bonadei, I.; Vizzardi, E.; Sciatti, E.; Lorusso, R. On-Pump Coronary Artery Bypass Graft: The State of the Art. Rev. Recent Clin. Trials 2019, 14, 106-115. [CrossRef] [PubMed]

2. Ong, C.S.; Zhou, X.; Huang, C.Y.; Fukunishi, T.; Zhang, H.; Hibino, N. Tissue engineered vascular grafts: Current state of the field. Expert Rev. Med. Devices 2017, 14, 383-392. [CrossRef] [PubMed]

3. Natasha, G.; Tan, A.; Gundogan, B.; Farhatnia, Y.; Nayyer, L.; Mahdibeiraghdar, S.; Rajadas, J.; De Coppi, P.; Davies, A.H.; Seifalian, A.M. Tissue engineering vascular grafts a fortiori: Looking back and going forward. Expert Opin. Biol. Ther. 2015, 15, 231-244. [CrossRef]

4. Hasan, A.; Memic, A.; Annabi, N.; Hossain, M.; Paul, A.; Dokmeci, M.R.; Dehghani, F.; Khademhosseini, A. Electrospun scaffolds for tissue engineering of vascular grafts. Acta Biomater. 2014, 10, 11-25. [CrossRef] [PubMed] 
5. Palumbo, V.D.; Bruno, A.; Tomasello, G.; Damiano, G.; Lo Monte, A.I. Bioengineered vascular scaffolds: The state of the art. Int. J. Artif. Organs. 2014, 37, 503-512. [CrossRef]

6. Rocco, K.A.; Maxfield, M.W.; Best, C.A.; Dean, E.W.; Breuer, C.K. In vivo applications of electrospun tissue-engineered vascular grafts: A review. Tissue Eng. Part B Rev. 2014, 20, 628-640. [CrossRef]

7. Song, H.G.; Rumma, R.T.; Ozaki, C.K.; Edelman, E.R.; Chen, C.S. Vascular Tissue Engineering: Progress, Challenges, and Clinical Promise. Cell Stem Cell 2018, 22, 340-354. [CrossRef]

8. Tara, S.; Rocco, K.A.; Hibino, N.; Sugiura, T.; Kurobe, H.; Breuer, C.K.; Shinoka, T. Vessel bioengineering. Circ. J. 2014, 78, 12-19. [CrossRef]

9. Dimitrievska, S.; Niklason, L.E. Historical Perspective and Future Direction of Blood Vessel Developments. Cold Spring Harb. Perspect. Med. 2018, 8, a025742. [CrossRef]

10. Radke, D.; Jia, W.; Sharma, D.; Fena, K.; Wang, G.; Goldman, J.; Zhao, F. Tissue Engineering at the Blood-Contacting Surface: A Review of Challenges and Strategies in Vascular Graft Development. Adv. Healthc. Mater. 2018, 7, e1701461. [CrossRef]

11. Ren, X.; Feng, Y.; Guo, J.; Wang, H.; Li, Q.; Yang, J.; Hao, X.; Lv, J.; Ma, N.; Li, W. Surface modification and endothelialization of biomaterials as potential scaffolds for vascular tissue engineering applications. Chem. Soc. Rev. 2015, 44, 5680-5742. [CrossRef] [PubMed]

12. Mead, L.E.; Prater, D.; Yoder, M.C.; Ingram, D.A. Isolation and characterization of endothelial progenitor cells from human blood. Curr. Protoc. Stem Cell Biol. 2008, 6, 2C.1.1-2C.1.27. [CrossRef] [PubMed]

13. Mund, J.A.; Estes, M.L.; Yoder, M.C.; Ingram, D.A., Jr.; Case, J. Flow cytometric identification and functional characterization of immature and mature circulating endothelial cells. Arterioscler. Thromb. Vasc. Biol. 2012, 32, 1045-1053. [CrossRef] [PubMed]

14. Matveeva, V.; Khanova, M.; Sardin, E.; Antonova, L.; Barbarash, O. Endovascular Interventions Permit Isolation of Endothelial Colony-Forming Cells from Peripheral Blood. Int. J. Mol. Sci. 2018, 19, 3453. [CrossRef] [PubMed]

15. Joo, H.J.; Song, S.; Seo, H.R.; Shin, J.H.; Choi, S.C.; Park, J.H.; Yu, C.W.; Hong, S.J.; Lim, D.S. Human endothelial colony forming cells from adult peripheral blood have enhanced sprouting angiogenic potential through up-regulating VEGFR2 signaling. Int. J. Cardiol. 2015, 197, 33-43. [CrossRef] [PubMed]

16. Yoder, M.C.; Mead, L.E.; Prater, D.; Krier, T.R.; Mroueh, K.N.; Li, F.; Krasich, R.; Temm, C.J.; Prchal, J.T.; Ingram, D.A. Redefining endothelial progenitor cells via clonal analysis and hematopoietic stem/progenitor cell principals. Blood 2007, 109, 1801-1809. [CrossRef]

17. Hauser, S.; Jung, F.; Pietzsch, J. Human Endothelial Cell Models in Biomaterial Research. Trends Biotechnol. 2017, 35, 265-277. [CrossRef]

18. Aird, W.C. Endothelial cell heterogeneity. Cold Spring Harb. Perspect. Med. 2012, 2, a006429. [CrossRef]

19. Dejana, E.; Hirschi, K.K.; Simons, M. The molecular basis of endothelial cell plasticity. Nat. Commun. 2017, 8, 14361. [CrossRef]

20. Campagnolo, P.; Tsai, T.N.; Hong, X.; Kirton, J.P.; So, P.W.; Margariti, A.; Di Bernardini, E.; Wong, M.M.; $\mathrm{Hu}$, Y.; Stevens, M.M.; et al. c-Kit+ progenitors generate vascular cells for tissue-engineered grafts through modulation of the Wnt/Klf4 pathway. Biomaterials 2015, 60, 53-61. [CrossRef]

21. Lin, R.Z.; Moreno-Luna, R.; Li, D.; Jaminet, S.C.; Greene, A.K.; Melero-Martin, J.M. Human endothelial colony-forming cells serve as trophic mediators for mesenchymal stem cell engraftment via paracrine signaling. Proc. Natl. Acad. Sci. USA 2014, 111, 10137-10142. [CrossRef]

22. Hibino, N.; Duncan, D.R.; Nalbandian, A.; Yi, T.; Qyang, Y.; Shinoka, T.; Breuer, C.K. Evaluation of the use of an induced puripotent stem cell sheet for the construction of tissue-engineered vascular grafts. $J$ Thorac. Cardiovasc. Surg. 2012, 143, 696-703. [CrossRef] [PubMed]

23. Kolbe, M.; Dohle, E.; Katerla, D.; Kirkpatrick, C.J.; Fuchs, S. Enrichment of outgrowth endothelial cells in high and low colony-forming cultures from peripheral blood progenitors. Tissue Eng. Part C Methods 2010, 16, 877-886. [CrossRef]

24. Jaffe, E.A.; Nachman, R.L.; Becker, C.G.; Minick, C.R. Culture of human endothelial cells derived from umbilical veins. Identification by morphologic and immunologic criteria. J. Clin. Investig. 1973, 52, 2745-2756. [CrossRef] [PubMed]

25. Ingram, D.A.; Mead, L.E.; Tanaka, H.; Meade, V.; Fenoglio, A.; Mortell, K.; Pollok, K.; Ferkowicz, M.J.; Gilley, D.; Yoder, M.C. Identification of a novel hierarchy of endothelial progenitor cells using human peripheral and umbilical cord blood. Blood 2004, 104, 2752-2760. [CrossRef] 
26. Liu, Y.; Teoh, S.H.; Chong, M.S.; Lee, E.S.; Mattar, C.N.; Randhawa, N.K.; Zhang, Z.Y.; Medina, R.J.; Kamm, R.D.; Fisk, N.M.; et al. Vasculogenic and osteogenesis-enhancing potential of human umbilical cord blood endothelial colony-forming cells. Stem Cells 2012, 30, 1911-1924. [CrossRef] [PubMed]

27. Denecke, B.; Horsch, L.D.; Radtke, S.; Fischer, J.C.; Horn, P.A.; Giebel, B. Human endothelial colony-forming cells expanded with an improved protocol are a useful endothelial cell source for scaffold-based tissue engineering. J. Tissue Eng. Regen. Med. 2015, 9, E84-E97. [CrossRef]

28. Rossi, E.; Poirault-Chassac, S.; Bieche, I.; Chocron, R.; Schnitzler, A.; Lokajczyk, A.; Bourdoncle, P.; Dizier, B.; Bacha, N.C.; Gendron, N.; et al. Human Endothelial Colony Forming Cells Express Intracellular CD133 that Modulates their Vasculogenic Properties. Stem Cell Rev. Rep. 2019, 15, 590-600. [CrossRef]

29. Rapp, B.M.; Saadatzedeh, M.R.; Ofstein, R.H.; Bhavsar, J.R.; Tempel, Z.S.; Moreno, O.; Morone, P.; Booth, D.A.; Traktuev, D.O.; Dalsing, M.C.; et al. Resident Endothelial Progenitor Cells From Human Placenta Have Greater Vasculogenic Potential Than Circulating Endothelial Progenitor Cells From Umbilical Cord Blood. Cell Med. 2011, 2, 85-96. [CrossRef]

30. Patel, J.; Seppanen, E.; Chong, M.S.; Yeo, J.S.; Teo, E.Y.; Chan, J.K.; Fisk, N.M.; Khosrotehrani, K. Prospective surface marker-based isolation and expansion of fetal endothelial colony-forming cells from human term placenta. Stem Cells Transl. Med. 2013, 2, 839-847. [CrossRef]

31. Solomon, I.; O’Reilly, M.; Ionescu, L.; Alphonse, R.S.; Rajabali, S.; Zhong, S.; Vadivel, A.; Shelley, W.C.; Yoder, M.C.; Thébaud, B. Functional Differences Between Placental Micro- and Macrovascular Endothelial Colony-Forming Cells. Stem Cells Transl. Med. 2016, 5, 291-300. [CrossRef] [PubMed]

32. Yu, S.; Li, Z.; Zhang, W.; Du, Z.; Liu, K.; Yang, D.; Gong, S. Isolation and characterization of endothelial colony-forming cells from mononuclear cells of rat bone marrow. Exp. Cell Res. 2018, 370, 116-126. [CrossRef]

33. Lin, R.Z.; Moreno-Luna, R.; Muñoz-Hernandez, R.; Li, D.; Jaminet, S.C.; Greene, A.K.; Melero-Martin, J.M. Human white adipose tissue vasculature contains endothelial colony-forming cells with robust in vivo vasculogenic potential. Angiogenesis 2013, 16, 735-744. [CrossRef] [PubMed]

34. Alphonse, R.S.; Vadivel, A.; Zhong, S.; McConaghy, S.; Ohls, R.; Yoder, M.C.; Thébaud, B. The isolation and culture of endothelial colony-forming cells from human and rat lungs. Nat. Protoc. 2015, 10, 1697-1708. [CrossRef] [PubMed]

35. Prasain, N.; Lee, M.R.; Vemula, S.; Meador, J.L.; Yoshimoto, M.; Ferkowicz, M.J.; Fett, A.; Gupta, M.; Rapp, B.M.; Saadatzadeh, M.R.; et al. Differentiation of human pluripotent stem cells to cells similar to cord-blood endothelial colony-forming cells. Nat. Biotechnol. 2014, 32, 1151-1157. [CrossRef] [PubMed]

36. Anderson, D.E.J.; Truong, K.P.; Hagen, M.W.; Yim, E.K.F.; Hinds, M.T. Biomimetic modification of poly(vinyl alcohol): Encouraging endothelialization and preventing thrombosis with antiplatelet monotherapy. Acta Biomater. 2019, 86, 291-299. [CrossRef]

37. Hammer, J.; Han, L.H.; Tong, X.; Yang, F. A facile method to fabricate hydrogels with microchannel-like porosity for tissue engineering. Tissue Eng. Part C Methods 2014, 20, 169-176. [CrossRef]

38. Athirasala, A.; Lins, F.; Tahayeri, A.; Hinds, M.; Smith, A.J.; Sedgley, C.; Ferracane, J.; Bertassoni, L.E. A Novel Strategy to Engineer Pre-Vascularized Full-Length Dental Pulp-like Tissue Constructs. Sci. Rep. 2017, 7, 3323. [CrossRef]

39. Rouwkema, J.; Khademhosseini, A. Vascularization and Angiogenesis in Tissue Engineering: Beyond Creating Static Networks. Trends Biotechnol. 2016, 34, 733-745. [CrossRef]

40. Szöke, K.; Reinisch, A.; Østrup, E.; Reinholt, F.P.; Brinchmann, J.E. Autologous cell sources in therapeutic vasculogenesis: In vitro and in vivo comparison of endothelial colony-forming cells from peripheral blood and endothelial cells isolated from adipose tissue. Cytotherapy 2016, 8, 242-252. [CrossRef]

41. Nuzzolo, E.R.; Capodimonti, S.; Martini, M.; Iachininoto, M.G.; Bianchi, M.; Cocomazzi, A.; Zini, G.; Leone, G.; Larocca, L.M.; Teofili, L. Adult and cord blood endothelial progenitor cells have different gene expression profiles and immunogenic potential. Blood Transfus. 2014, 12 (Suppl. 1), s367-s374. [CrossRef] [PubMed]

42. Zeisberger, S.M.; Zoller, S.; Riegel, M.; Chen, S.; Krenning, G.; Harmsen, M.C.; Sachinidis, A.; Zisch, A.H. Optimization of the culturing conditions of human umbilical cord blood-derived endothelial colony-forming cells under xeno-free conditions applying a transcriptomic approach. Genes Cells 2010, 15, 671-687. [CrossRef] [PubMed]

43. Moccia, F.; Fotia, V.; Tancredi, R.; Della Porta, M.G.; Rosti, V.; Bonetti, E.; Poletto, V.; Marchini, S.; Beltrame, L.; Gallizzi, G.; et al. Breast and renal cancer-derived endothelial colony forming cells share a common gene signature. Eur. J. Cancer 2017, 77, 155-164. [CrossRef] [PubMed] 
44. Simoncini, S.; Chateau, A.L.; Robert, S.; Todorova, D.; Yzydorzick, C.; Lacroix, R.; Ligi, I.; Louis, L.; Bachelier, R.; Simeoni, U.; et al. Biogenesis of Pro-senescent Microparticles by Endothelial Colony Forming Cells from Premature Neonates is driven by SIRT1-Dependent Epigenetic Regulation of MKK6. Sci. Rep. 2017, 7, 8277. [CrossRef]

45. Shafiee, A.; Patel, J.; Wong, H.Y.; Donovan, P.; Hutmacher, D.W.; Fisk, N.M.; Khosrotehrani, K. Priming of endothelial colony-forming cells in a mesenchymal niche improves engraftment and vasculogenic potential by initiating mesenchymal transition orchestrated by NOTCH signaling. FASEB J. 2017, 31, 610-624. [CrossRef]

46. Kovacic, J.C.; Dimmeler, S.; Harvey, R.P.; Finkel, T.; Aikawa, E.; Krenning, G.; Baker, A.H. Endothelial to Mesenchymal Transition in Cardiovascular Disease: JACC State-of-the-Art Review. J. Am. Coll. Cardiol. 2019, 73, 190-209. [CrossRef]

47. Li, Y.; Lui, K.O.; Zhou, B. Reassessing endothelial-to-mesenchymal transition in cardiovascular diseases. Nat Rev Cardiol. 2018, 15, 445-456. [CrossRef]

48. Souilhol, C.; Harmsen, M.C.; Evans, P.C.; Krenning, G. Endothelial-mesenchymal transition in atherosclerosis. Cardiovasc. Res. 2018, 114, 565-577. [CrossRef]

49. DiMaio, T.A.; Wentz, B.L.; Lagunoff, M. Isolation and characterization of circulating lymphatic endothelial colony forming cells. Exp. Cell Res. 2016, 340, 159-169. [CrossRef]

50. Schneller, D.; Hofer-Warbinek, R.; Sturtzel, C.; Lipnik, K.; Gencelli, B.; Seltenhammer, M.; Wen, M.; Testori, J.; Bilban, M.; Borowski, A.; et al. Cytokine-Like 1 Is a Novel Proangiogenic Factor Secreted by and Mediating Functions of Endothelial Progenitor Cells. Circ. Res. 2019, 124, 243-255. [CrossRef]

(C) 2020 by the authors. Licensee MDPI, Basel, Switzerland. This article is an open access article distributed under the terms and conditions of the Creative Commons Attribution (CC BY) license (http://creativecommons.org/licenses/by/4.0/). 